\title{
Alkali-hydrogen reactions
}

\author{
KING-CHUEN LIN
}

Department of Chemistry, National Taiwan University, and Institute of Atomic and Molecular Sciences, Academia Sinica, Taipei 106, Taiwan

\section{RAYMOND VETTER}

Laboratoire Aimé Cotton, Centre National de la Recherche Scientifique, Bâtiment 505, Campus d'Orsay, 91405 Orsay Cedex, France

Highly endoergic alkali-hydrogen reactions are usually initiated by electronic excitation of the alkali atom. This implies that non-adiabatic couplings take place along the reaction path, the collision mechanism being dominated by the electron transfer jump that occurs between the alkali atom and the hydrogen molecule. Since electronic excitation can be selectively achieved over many atomic states, these reactions constitute good candidates to generalize the study of non-adiabatic processes in reaction dynamics. Series of heat-pipe oven and crossed-beam experiments have been carried out over the $\mathrm{Li}+\mathrm{H}_{2}, \mathrm{Na}+\mathrm{H}_{2}, \mathrm{~K}+\mathrm{H}_{2}, \mathrm{Rb}+\mathrm{H}_{2}$ and $\mathrm{Cs}+\mathrm{H}_{2}$ reactions, in which high-resolution optical techniques have been used for reagent excitation and product characterization. The experimental findings indicate that, in spite of common characteristics, the five reactions exhibit apparently different behaviours that are not explained by usual collision models. However, a critical analysis based on the few calculations of ab initio potential energy surfaces and collision dynamics shows that the electronic symmetry of the reagent-product system plays a major role in the reaction, and not the potential energy above threshold. The way in which the electron transfer occurs along the reaction path is the key factor that influences the collision mechanism: side-on insertion for lithium and sodium and collinear abstraction for potassium, rubidium and caesium. The height of the potential barrier and the size and excitation energy of the alkali atom are also deciding factors. More systematic studies and new experiments are necessary to confirm these trends and to complete our understanding of these model reactions.

\section{Contents}

2. Reaction dynamics: experimental and theoretical findings

3. Alkali-hydrogen reactions: the state of the art

3.1. $\mathrm{Li}+\mathrm{H}_{2}$

3.2. $\mathrm{Na}+\mathrm{H}_{2}$

3.3. $\mathrm{K}+\mathrm{H}_{2}$

3.4. $\mathrm{Rb}+\mathrm{H}_{2}$

3.5. $\mathrm{Cs}+\mathrm{H}_{2}$ 
4. Alkali-hydrogen reactions: the general trends

\section{Conclusions}

Acknowledgements

\section{Introduction}

The aim of reaction dynamics studies is to determine the basic fundamental mechanisms of chemical reactivity and eventually to influence the route of the chemical reaction to yield highly selected products. Experimentally, this is achieved in the gas phase at the most elementary level of individual isolated collisions, from the control of reagent physical parameters and the probe of reaction products in their nascent states. Theoretically, the aim is to calculate the potential energy of the reactive system and to evaluate its collision dynamics. From the experiments and calculations performed for several decades, the main features of reaction dynamics were observed and led to realistic collision models, mostly for simple reactive systems in their ground state (Fluendy and Lawley 1973, Child 1974, Polanyi and Schreiber 1974, Toennies 1974, Smith 1980, Bernstein 1982,1984, Levine and Bernstein 1987, Scoles 1988, Gonzales-Urena and Vetter 1996).

The advent of lasers greatly enlarged the field of reaction dynamics studies, through the selective electronic excitation of reagents and the high-resolution characterization of product states (Bergmann 1988, Demtröder 1988, Hefter and Bergmann 1988, Gonzales-Urena and Vetter 1995, 1996). Furthermore, new experiments taking the particular nature of laser light into account were carried out to extend the understanding of collision dynamics (Bernstein 1982, Scoles 1988, Gonzales-Urena and Vetter 1995, 1996). However, in spite of these developments, the detailed comparison between theory and experiment is still limited to several elementary reactions in their ground states, among which the $\mathrm{H}+\mathrm{D}_{2} \rightarrow \mathrm{HD}+\mathrm{D}$ and $\mathrm{F}+\mathrm{H}_{2} \rightarrow \mathrm{FH}+\mathrm{H}$ reactions are the most popular (Polanyi and Woodall 1972, Gerrity and Valentini 1983, 1985, Marinero et al. 1984, Neumark et al. 1985, Veirs et al. 1985, Rinnen et al. 1989].

In this field of research, the reactions between alkali atoms and molecular hydrogen, $\mathrm{A}+\mathrm{H}_{2} \rightarrow \mathrm{AH}+\mathrm{H}$, offer a number of interesting characteristics. The most important is certainly the fact that they are highly endoergic in their ground state, requiring a large deposit of energy to initiate the reaction. If in principle this deposit can be given in the form of vibrational and/or kinetic energy, in practice it is easily achieved via electronic excitation of the atom. Then, as the $\mathrm{A}+\mathrm{H}_{2}$ groundstate potential surface is adiabatically correlated to the $\mathrm{AH}+\mathrm{H}$ ground state one, non-adiabatic couplings necessarily occur along the photochemical reaction path, from the excited entrance valley to the exit valley. As electronic excitation of the atom is usually achieved by using lasers, it opens the way to the study of various selective effects such as the influence of potential energy and electronic symmetry, as well as the influence of light polarization and coherence. The second common characteristic lies in the limited number of atoms and active electrons that are involved in the reaction, allowing for realistic accurate calculations of potential energy surfaces (PESs) and collision dynamics, even in the excited states that are 
concerned. Since alkali hydrides absorb light in the visible, they constitute good candidates for applying high-resolution optical techniques such as laser-induced fluorescence or resonance-enhanced multiphoton ionization to the characterization of reaction products. Therefore, these photochemical reactions offer unique possibilities for detailed generalized studies of non-adiabatic processes in reaction dynamics. Until now, there have been no other types of reaction that can lead to such a generalization.

The five photochemical reactions involving lithium, sodium, potassium, rubidium and caesium have been studied by use of various experimental and theoretical techniques that lead to apparently contradictory results whose clarification is the subject of this review. Accordingly, the article is organized as follows. Experimental and theoretical techniques are briefly reviewed in section 2 to determine what is expected from reaction dynamics studies in the gas phase. The results obtained over the five reactions are described in section 3 and their common features are analysed in section 4. Conclusions, involving a few future directions of research, are drawn in section 5 .

\section{Reaction dynamics: experimental and theoretical findings}

The experimental objective is to define as exactly as possible the various physical parameters that prepare the reactive collision (all the forms of energy, electronic symmetry, geometry of approach, etc.) and to characterize the products in their nascent states (outcomes, distributions of energy and angular scattering, etc.). These experimental findings require that the so-called 'single-collision' conditions be fulfilled, with no post-reaction redistribution by collision. There is no way here to follow the reactive collision in real time, an experiment that is still out of reach in spite of the development of femtosecond lasers, but to define the reactive system as well as possible 'before' and 'after' the reactive collision.

What is actually measurable in reaction dynamics?

The first scalar quantity is the total cross section $\sigma_{\mathrm{R}}\left(E_{\mathrm{c}}\right)$ that characterizes the efficiency of the overall process at a given reagent kinetic energy $E_{\mathrm{c}}$. For a given $\mathrm{A}+\mathrm{BC} \rightarrow \mathrm{AB}+\mathrm{C}$ reaction studied under single-collision conditions, it is defined by (Levine and Bernstein 1987, Gonzales-Urena and Vetter 1993,1996):

$$
\sigma_{\mathrm{R}}\left(E_{\mathrm{c}}\right)=\frac{\mathrm{d} N_{\mathrm{AB}}}{\mathrm{d} t}\left(n_{\mathrm{A}} n_{\mathrm{BC}} V_{\mathrm{r}} \Delta V\right)^{-1},
$$

in which $\mathrm{d} N_{\mathrm{AB}} / \mathrm{d} t$ is the total number of $\mathrm{AB}$ molecules created per second at the collision volume $\Delta V$ (or the total flux $F_{\mathrm{AB}}$ ), the $n$ values are the particle densities and $V_{\mathrm{r}}$ is the relative velocity. Typical reactive cross-sections range between 0.01 and $100 \AA^{2}$. The total cross-section is linked to the usual reaction rate constant by averaging over a Maxwell-Boltzmann reagent velocity distribution, namely

$$
k(T)=\left(\pi_{\mu}\right)^{-1 / 2}\left(\frac{2}{k_{\mathrm{B}} T}\right)^{3 / 2} \int \sigma_{\mathrm{R}}\left(E_{\mathrm{c}}\right) \exp \left(\frac{-E_{\mathrm{c}}}{k_{\mathrm{B}} T}\right) \mathrm{d} E_{\mathrm{T}}
$$

in which $\mu$ is the reduced mass of the reagents, $k_{\mathrm{B}}$ is the Boltzmann constant and $T$ is the temperature. This equation, which holds if $\sigma_{\mathrm{R}}\left(E_{\mathrm{c}}\right)$ is a function of $E_{\mathrm{c}}$ only, is the link between microscopic dynamics and macroscopic kinetics.

It is usual to define other scalar quantities, whose characterization depends upon the degree of sophistication of the experiment. For instance, the 'state-to-state' cross- 
section measures the efficiency of the reactive process from a well-defined reagent quantum state to a well-defined product state. By summing over all reagent and product states of this 'differential' cross-section, one recovers the previous total cross-section. The 'excitation function' describes the variation in the total crosssection versus the reagent kinetic energy and shows the existence of potential barriers on the reaction path, if any. Product states are characterized by their energy partitioning, that is the kinetic-electronic-vibrational-rotational distributions that result from the averaging of state-to-state cross-sections over reagent states.

The reactive process depends also upon vectorial quantities, such as the impact parameter and the reagent's angle of approach. The impact parameter is still out of reach, even in the most sophisticated experiment, and the angle of approach can be controlled for the molecules that possess large dipole moments only. However, crossed-beam experiments have successfully demonstrated that reaction products scatter at various angles with respect to the relative velocity vector. Accordingly, the flux of $\mathrm{AB}$ particles scattered per unit time per unit solid angle in the centre-of-mass framework is

$$
F_{\mathrm{AB}}(\theta, \phi)=n_{\mathrm{A}} n_{\mathrm{BC}} V_{\mathrm{r}} \Delta V \sigma_{\mathrm{R}} P(\theta, \phi) .
$$

$\sigma_{\mathrm{R}} P(\theta, \phi)$ is the differential (solid-angle) cross-section, $P(\theta, \phi)$ being the probability density that products have to scatter at the angle $(\theta, \phi)$ with respect to the relative velocity vector. If there is no azimuthal dependence of the scattering, that is the collision trajectory is confined to a plane, the total cross section is

$$
\sigma_{\mathrm{R}}=2 \pi \int_{0}^{\pi} \sigma_{\mathrm{R}} P(\theta) \sin \theta \mathrm{d} \theta .
$$

$P(\theta)$ is the (normalized) probability density that products have to scatter at the angle $\theta$ with respect to the relative velocity vector. An important feature is that the shape of $P(\theta)$ may be related to the reaction mechanism. For instance, the 'harpooning' mechanism that is relevant for direct reactions between alkalis and halogens gives rise to 'forward' scattering in the centre-of-mass framework because the reactive collision takes place at large internuclear distances; on the contrary, the 'rebound' mechanism that is relevant for reactions between alkalis and methyl iodide gives rise to 'backward' scattering; an indirect reaction with the formation of a long-lived intermediate complex leads to isotropic scattering in the centre-of-mass framework. Since it is now possible to orientate molecular reagents with respect to the direction of approach, at least under particular experimental conditions, deep insights into the stereodynamics of the reaction are expected from these findings.

The previous examples show the variety of parameters that one wants to control to obtain a maximum of data about the collision process. The 'ideal' experiment still does not exist, but many of them approach particular aspects of the problem. Lowpressure gas cell or gas-flow reactor experiments were historically the first to demonstrate the basic features of elementary reactions. Results carried out in the 1930s by Polanyi (1932) about alkali-halogen reactions that led to the concept of harpooning should be mentioned. Later, the development of crossed-beam experiments by Herschbach $(1965,1966,1987)$ revealed the vectorial nature of the reactive process. More recently, the use of lasers opened up the field to more sophisticated experiments whose review is outside the scope of this article. In the restricted field of alkali-hydrogen reactions, two types of experiment are currently carried out, which are briefly described in the following. 
In heat-pipe oven experiments, a five- or six-armed stainless steel cylinder cell is filled with alkali metal and molecular hydrogen at a low pressure, several torrs typically. It is heated at several hundred degrees Celsius to provide a low but substantial partial pressure of the alkali. The endoergic reaction is initiated by electronic excitation of the atom, by use of a pulsed laser beam whose frequency is locked to a resonant atomic absorption line. Reaction products are probed by a second laser beam whose pulse is delayed with respect to the first beam, the delay being shorter than the mean time between relaxation collisions, that is, reaction products are probed in their nascent states ('pump-probe' technique). The experiment is well adapted to study the influence of potential energy and electronic symmetry over reactivity since the electronic excitation can be easily carried out over many quantum states of the atom, including its fine structure. In the bulk, velocity distributions are typically Maxwell-Boltzmann without privileged direction or magnitude, meaning that there is no way to determine angular and kinetic distributions and also that partial distributions are thermally averaged. However, in this framework, the determination of product rovibrational distributions is always possible. By analogy with equation (1), the rate of product formation is

$$
\frac{\mathrm{d}[C]}{\mathrm{d} t}=\sigma_{\mathrm{R}} V_{\mathrm{r}}[\mathrm{A}][\mathrm{BC}] \text {. }
$$

The first application of the pump-probe technique is due to Breckenridge and Umemoto who studied the $\mathrm{Mg}\left(3 \mathrm{p}{ }^{1} \mathrm{P}\right)+\mathrm{H}_{2} \rightarrow \mathrm{MgH}+\mathrm{H}$ reaction. They observed that the rotational state distribution of $\mathrm{MgH}$ is bimodal and they concluded that the reaction proceeds through two microscopic pathways (Breckenridge and Umemoto 1981, 1984). Many other applications deal with alkali-hydrogen reactions (Lin and Chang 1989, Bililign and Kleiber 1990, Bililign et al. 1992, Huang et al. 1996, Fan et al. 1999). It should be mentioned that some refinements of the technique were developed to control a few parameters of the reactive collision better (Hering et al. 1980, Tsukiyama et al. 1980, Scherer et al. 1987, Brouard et al. 1991, 1992, Van der Zande et al. 1991); unfortunately they concern particular well-adapted reactions.

An inherent difficulty linked to this type of experiment lies in the competition between highly excited states to yield the same reaction products since, once an electronic atomic state is excited, its population may flow into lower states through collision relaxations and radiation cascades. A quantitative measure of such a product interference from other excited states was done in the $\operatorname{Mg}\left(4 \mathrm{~s}{ }^{1} \mathrm{~S}_{0}, 3 \mathrm{~d}\right.$ $\left.{ }^{1} \mathrm{D}_{2}\right)+\mathrm{H}_{2}$ reactions by looking at the influence of the lower $3 \mathrm{p}{ }^{1} \mathrm{P}_{1}$ state (Liu et al. 2000). This was realized by use of a third pulsed laser beam to deplete the $3 p{ }^{1} \mathrm{P}_{1}$ population by exciting the $3 \mathrm{p}^{1} \mathrm{P}_{1} \rightarrow 6 \mathrm{~d}{ }^{1} \mathrm{D}_{2}$ transition. A substantial fraction of the $3 p{ }^{1} P_{1}$ population was pumped out to the $6 d{ }^{1} D_{2}$ state whose lifetime is longer than the time needed for $\mathrm{MgH}$ formation, so that increasing the intensity of the depletion laser decreased the yield of $\mathrm{MgH}$. The influence of the $3 \mathrm{p}^{1} \mathrm{P}_{1}$ population on the $\mathrm{MgH}$ product distribution was thus determined directly without involving any complicated evaluation.

In crossed-beam experiments, two beams of particles cross at a right angle in a low-pressure collision chamber, a laser beam being used to excite the alkali atom at the cross volume and to initiate the endoergic reaction. According to the low densities of particles, the conditions for single collisions are automatically fulfilled. Optimized conditions are obtained if supersonic expansions are used for the reagent molecular beams because they lead to a drastic reduction in the velocity spreads and 
to condensation of ground-state molecules in their lowest rovibrational states. Thus, this technique opens the way to 'state-to-state' studies of reaction dynamics. In addition, the use of seeding techniques allows the reagent kinetic energy to be varied, and hence the excitation function and the height of potential barriers in the entrance valley of the reaction path, if any. Usual measurements by mass spectrometry, timeof-flight and high-resolution optical techniques involve the determination of translational and rovibrational product state distributions but the main advantage of the experiment is to provide the differential angular cross section. This is achieved either by rotating a mass spectrometer around the cross volume (Valentini et al. 1977) or by using a second laser beam to probe reaction products at the cross volume through the so-called 'Doppler technique' (Kinsey 1977, Murphy et al. 1979, Serri et al. 1981, L'Hermite et al. 1990a, b, Mestdagh et al. 1994). The experiment is well adapted to study the influence of alignment and orientation of atomic and molecular reagents over the reaction yield, and generally speaking to study anisotropy in reaction dynamics.

Many crossed-beam experiments have been developed in the past to demonstrate the main features of reaction dynamics, and the anisotropic character of product scattering in particular. Bench-mark studies were carried out by many groups (Herschbach 1965, 1966, 1987, Kramer and Bernstein 1965, Grice and Empedocles 1968, Gillen et al. 1971, Rulis and Bernstein 1972, Zare 1972, 1977, 1979, Bernstein and Rulis 1973, Cruse et al. 1973, Parson et al. 1973, Valentini et al. 1977, Becker et al. 1980, Mayne and Toennies 1981, Parker et al. 1982, Stolte et al. 1982, Gotting et al. 1984, 1986, Neumark et al. 1985a, b, Bernstein 1986, Gandhi et al. 1986, Weiss et al. 1986, Vernon et al. 1986, Lee 1987) (this list is not exhaustive of course!). The pioneering experiments in which the polarized nature of the laser light was used to align atomic orbitals with respect to the collision axis should also be mentioned (Rettner and Zare 1981, 1982, Leone 1988, Menzinger 1988, Davies et al. 1990) and also the most refined experiments in which the 'brute force' technique was used to orientate molecular reagents with respect to the collision axis, this technique being limited to reagents with high permanent dipole moments, unfortunately (Loesch and Reincheid 1990, 1991, Freidrich and Herschbach 1991). Only one crossed-beam setup was devoted to the study of alkali-hydrogen reactions [Crépin et al. 1984, Rahmat et al. 1986, 1987, Gadéa et al. 1988, L'Hermite et al. 1990a, b, 1991, Cavero et al. 1999).

From the theoretical point of view, the aim is to build PESs and, once they are accurately described, to calculate the collision dynamics. The principal difficulty is linked to the formidable number of degrees of freedom that is involved to calculate potentials, even for small reactive systems in their ground states. Although the problem is theoretically solved for three-body systems, many efforts were necessary to yield reliable potentials before semiclassical and quantum calculations of the dynamics could be in agreement with experiment. In fact, for the simplest $\mathrm{H}+\mathrm{D}_{2} \rightarrow \mathrm{HD}+\mathrm{D}$ exchange reaction, experimental results are difficult to obtain owing to the weakness of the reactive process and to the hardness of product state characterization (Tsukiyama et al. 1980, Blais and Truhlar 1983, Gerrity and Valentini 1983,1985, Marinero et al. 1984, Meier et al. 1985, Veirs et al. 1985, Nieh and Valentini 1988, 1990, Rinnen et al. 1989). Until now, there have been still a limited number of elementary systems for which one has both extended highresolution experimental data and reliable theoretical calculations. 
It is not within the scope of this review to detail the calculations of PESs by semiempirical or $a b$ initio techniques. The reader is referred to the books by Schaefer (1977a,b) and Hirst (1985) and to the book by Bernstein (1984) in which various specialized authors describe the approach and accuracy of such calculations. However, it should just be mentioned that a majority of the PESs obtained up to now concern adiabatic ground-state reactions. A large amount of work is still to be carried out for excited-state reactions, precisely those involving alkalis and hydrogen.

For alkali-hydrogen photochemical reactions, neutral and ionic PESs are involved because alkali hydrides present a strong ionic character in their ground states. They reveal the importance of charge transfers in the reaction processes, as they do occur in the harpooning mechanism (Polanyi and Woodall 1972, Bernstein 1982, Levine and Bernstein 1987). In this process that typically holds for alkalihalogen reactions, $\mathrm{A}+\mathrm{X}_{2} \rightarrow \mathrm{AX}+\mathrm{X}$, the optical electron of the alkali is suddenly thrown as a harpoon to the halogen molecule; hence Coulombic attraction occurs between $\mathrm{A}^{+}$and $\mathrm{X}_{2}^{-}$particles, and then formation of the $\mathrm{A}^{+}-\mathrm{X}_{2}^{-}$intermediate followed by dissociation into $\mathrm{A}^{+} \mathrm{X}^{-}$and $\mathrm{X}$ products. In terms of potentials, the $\mathrm{A}+\mathrm{X}_{2}$ system propagates along the covalent entrance valley and jumps onto the ionic $\mathrm{A}^{+}-\mathrm{X}_{2}^{-}$surface which further leads to the exit valley of the products. The interatomic distance $R$ corresponding to the electron transfer is the distance at which the covalent and ionic surfaces cross, that is the effective bond length of the ion pair. It is easily evaluated by writing the equality between the Coulombic potential, the electron affinity (EA) of the halogen molecule and the ionization potential (IP) of the alkali atom (Polanyi and Woodall 1972, Bernstein 1982, Levine and Bernstein 1987):

$$
-\frac{e^{2}}{R} \approx-\mathrm{EA}+\mathrm{IP}
$$

Crossing distances are long for alkali-halogen reactions, up to $10 \AA$. As the probability of transfer from neutral to ionic is close to one, the corresponding reaction cross-sections $\sigma_{\mathrm{R}}=\pi R^{2}$ are easily up to several hundreds of square ångstroms. A pronounced 'forward' peaking of products in the centre-of-mass framework is generally associated with these direct reactions occurring at large impact parameters.

Based on the harpooning model, it has long been thought that the product energy partitioning into different degrees of freedom could be rationalized. A few collision models were developed to describe the energy partitioning in $\mathrm{A}+\mathrm{BC} \rightarrow \mathrm{AB}+\mathrm{C}$ reactions. The direct interaction with product repulsion model (DIPR) (Kuntz et al. 1969a, b, Prisant et al. 1984) assumes that the electron jump occurs at relatively large distances between $\mathrm{A}$ and $\mathrm{BC}$, thereby initiating a strong repulsive interaction in $\mathrm{BC}^{-}$ followed by an attractive interaction between $\mathrm{A}^{+}$and $\mathrm{B}^{-}$. Repulsive and attractive energy releases are considered to be separable. Accordingly, the repulsive energy release from the $\mathrm{BC}^{-}$bond rupture does not depend on the electron jump distance, which is essentially determined by the excitation energy. The impulsive model (Busch and Wilson 1972, Perry and Polanyi 1976, Schinke 1989) has sometimes provided a satisfactory explanation for product rotational and vibrational state distributions, but its reliability is questionable without support of PES information. In this model, the breaking apart of the single atom and the remaining diatomic molecule depends upon the initial bond angle only. It ignores the variation in the bond angle along the reaction coordinate and oversimplifies the treatment of potential interaction by using 
a diatomic potential term instead of a real PES. For example, in the $\operatorname{Mg}(3 \mathrm{p}$ $\left.{ }^{1} \mathrm{P}_{1}\right)+\mathrm{H}_{2}$ reaction, $\mathrm{MgH}$ products exhibit a bimodal rotational state distribution, with a major high rotational component and a minor low rotational component (Breckenridge and Umemoto 1981, 1984). Both are commonly caused by an insertion mechanism, the subsequent microscopic pathways being due to the extent of the anisotropic interaction in the exit channel. However, according to the impulsive model that neglects the variation in the bond angle, only the high rotational component is anticipated to result from the fragmentation of the bent $\mathrm{Mg}-\mathrm{H}_{2}$ intermediate. It is assumed here that the restoring force is exerted along the $\mathrm{H}-\mathrm{H}$ coordinate, the magnesium atom being treated as a spectator that does not take part in the dissociation process. Therefore, for this reaction, the model turns out to be contrary to the experimental findings.

Kuntz et al. (1969a, b) have thoroughly investigated the harpooning-type reaction by use of a classical trajectory method over an appropriate ionic potential surface. The resultant product energy distribution among various degrees of freedom was found to depend on the electron jump distance, the repulsive force for breaking the $\mathrm{X}_{2}^{-}$bond, the incident collision angle, the initial kinetic energy and the mass effect. In $\mathrm{L}+\mathrm{HH} \rightarrow \mathrm{LH}+\mathrm{H}$ reactions (L, light atom; H, heavy atom) in a collinear geometry of approach, the new LH bond tends to be formed before the $\mathrm{HH}$ repulsion completely dissipates. By contrast, in $\mathrm{H}+\mathrm{LL} \rightarrow \mathrm{HL}+\mathrm{L}$ reactions (e.g. alkali-hydrogen reactions) the bond forming and bond breaking occur simultaneously. To characterize the product energy partitioning in these reactions, it is thus appropriate to take into account such a 'mixed energy release', that is the release of LL repulsive energy while the new bond HL is still forming. The resulting coupling between repulsive and attractive energies exhibits two major features: firstly, the increase in repulsion may enhance the available energy partitioning into translation and, secondly, the fraction of repulsive energy release that goes to vibration increases with increasing atomic masses.

The following equation describes how the rotational state distribution depends upon the anisotropy of the potential in its exit channel. For a three-body system, for example $\mathrm{M}+\mathrm{H}_{2}$ reactions, one has in the Jacobi coordinates (Huber and Schinke 1993, Schinke 1993)

$$
\frac{\mathrm{d} \mathbf{j}}{\mathrm{d} t}=-\frac{\partial V(\theta, \mathbf{R})}{\partial \theta}
$$

in which $\mathbf{j}$ is the rotational angular momentum, $\mathbf{R}$ the distance between the departing hydrogen atom and the centre of mass of the $\mathrm{MH}$ product, $\mathbf{r}$ the bond length of $\mathrm{MH}$ and $\theta$ the Jacobi angle between $\mathbf{R}$ and $\mathbf{r}$ axes. According to this equation, the variation in the rotational angular momentum with time is related to the torque generated on the exit-channel surface. The supply of PES information is thus necessary to understand the microscopic branching for product state distributions.

This is not enough since a quantitative analysis still demands dynamics calculations. Among various sophisticated methods, quasiclassical trajectory (QCT) calculations have been popularly adopted for three-body systems (Bernstein 1984). They are carried out over analytic potential energy functions, fitted from $a b$ initio PESs. For an $\mathrm{M}+\mathrm{H}_{2}$ reaction, the initial conditions including the collision energy, the impact parameter and, for the hydrogen molecule, the initial hydrogen energy, the azimuthal and polar orientation angles of the internuclear axis, the 
orientation of the angular momentum and the phase angle of the vibration should be set up. Unless selective data are given, a random number generator is generally used to sample these conditions. The trajectories are initiated at a given distance between $\mathrm{M}$ and $\mathrm{H}_{2}$ and evolve at a small step (e.g. $1 \times 10^{-17} \mathrm{~s}$ ) to achieve energy conservation. When a trajectory computation is completed, slicing up the continuous distributions of final vibrational and rotational energies contained in a given $(v, J)$ quantum state of $\mathrm{MH}$ is obtained via the following rule: if the obtained classical energies are within half-way of the quantified energy gaps from the closest quantum state, then the portion of energy is assigned to this particular state.

Based on trajectory calculations, the opacity function, that is the reaction probability versus the impact parameter $b$ is evaluated by (Truhlar and Muckerman 1979)

$$
P(E, b)=\frac{N_{\mathrm{R}}(E, b)}{N_{\mathrm{T}}(E, b)}
$$

in which $N_{\mathrm{R}}(E, b)$ is the number of reactive trajectories with energy $E$ and impact parameter $b$ within the interval $[b, b+d b]$ and $N_{\mathrm{T}}(E, b)$ is the total number of such trajectories. The reaction cross section is therefore calculated by

$$
\sigma \mathrm{R}=2 \pi \int_{0}^{b_{\max }} P(b) b \mathrm{~d} b
$$

in which $b_{\max }$ is the upper limit of the impact parameter at which the collision does not lead to reaction. The product angular distribution in the centre-of-mass framework may be obtained by estimating the number of reactive trajectories that scatter at the angle $\theta$ in a given interval (e.g. $10^{\circ}$ ) out of the total trajectories scattered in this interval. The angular distribution obtained in this way is related to the differential cross section multiplied by $\sin \theta$. Many dynamics parameters such as reaction rate constants, rotational and vibrational state distributions are provided by QCT calculations, as well as vectorial information such as the correlation between reagent and product angular momenta (Zare 1988). Purely quantal techniques have also been used to calculate the dynamics of triatomic systems (Kuppermann 1981, Launay et al. 1986) but, for alkali-hydrogen reactions, the only test has so far concerned the $\mathrm{Cs}+\mathrm{H}_{2}$ reaction in a collinear geometry of approach [Lepetit et al. 1989).

For alkali-hydrogen photochemical reactions, the previous collision models should hold because the excited covalent entrance valley indeed crosses the $\mathrm{A}^{+}-$ $\mathrm{H}_{2}^{-}$ionic state at medium internuclear distances. However, for these reactions that are initiated by electronic excitation of the atom, the electron transfer occurs through a non-adiabatic process associated with the compulsory change of electronic symmetry. This leads to a reduced efficiency for the electron transfer and a smaller cross-section. Furthermore, it has been shown recently by ab initio calculations over a few alkali-hydrogen and alkaline-earth-hydrogen reactions that this electron transfer is not sudden but rather progressive (Jeung et al. 2002, Kim et al. 2002). In addition, a few experimental results have shown that the product energy partitioning does not systematically follow the rules predicted by the previous collision models. The concept of harpooning is thus questionable for these reactions. 


\section{Alkali-hydrogen reactions: the state of the art}

\section{1. $\mathrm{Li}+\mathrm{H}_{2}$}

The $\mathrm{Li}+\mathrm{H}_{2} \rightarrow \mathrm{LiH}+\mathrm{H}$ reaction is the simplest chemical reaction after the $\mathrm{H}+\mathrm{D}_{2}$ exchange reaction. It involves only three light atoms and five electrons in total, among which the weakly bound optical electron of the alkali atom is expected to play a major role. The reaction is endoergic by $2.05 \mathrm{eV}$ in its ground state $\dagger$ and is still endoergic by $0.20 \mathrm{eV}$ after excitation of lithium atoms to the $2 \mathrm{p}{ }^{2} \mathrm{P}$ state. It becomes largely exoergic after excitation to the $3 \mathrm{~s}{ }^{2} \mathrm{~S}$ state.

These features explain why only a few fragmentary experimental results are available for this system, in spite of interest. Indeed, if a supplementary amount of energy is deposited into the initial $\mathrm{Li}\left(2 \mathrm{p}^{2} \mathrm{P}\right)+\mathrm{H}_{2}$ system, the reaction can start from the first excited potential surface above ground state to yield $\mathrm{LiH}$ and $\mathrm{H}$ products in their electronic ground states. It necessarily implies non-adiabatic couplings along the reaction path, making it an ideal model to study non-adiabatic processes in reaction dynamics. Under crossed-beam conditions, the only clean selective way to deposit this supplementary amount of energy is to heat the hydrogen beam to $2000 \mathrm{~K}$, a sophisticated and dangerous technique that needs drastic precautionary measures. Under bulk conditions, high-energy collisions (in the tail of the MaxwellBoltzmann distribution) automatically fulfil the energy balance of the reaction, but they have a weak probability of occurrence. A second experimental difficulty is linked to the characterization of $\mathrm{LiH}$ products: by optical techniques because $\mathrm{LiH}$ molecules in their electronic ground state absorb light in the near ultraviolet, and by mass spectrometry because ionization of $\mathrm{LiH}$ molecules is accompanied by dissociation.

Until now, the only available experimental data over the $\operatorname{Li}\left(2 \mathrm{p}^{2} \mathrm{P}\right)+$ $\mathrm{H}_{2} \rightarrow \mathrm{LiH}+\mathrm{H}$ reaction have been obtained in bulk experiments. The first led to the measurement of the reaction cross-section $\left(\sigma=0.10 \AA^{2}\right)$ using a rate equation model (Mysers et al. 1987). The second led to the determination of the rotational state distribution in the $\mathrm{X}^{1} \Sigma^{+}\left(v^{\prime \prime}=0\right)$ level of $\mathrm{LiH}$, with a unimodal statistical distribution corresponding to a temperature of $770 \mathrm{~K}$, slightly hotter than the cell itself (figure 1) (Chen et al. 2001). It thus suggests a side-on attack of the lithium atom to insert between the two hydrogen atoms. By varying the temperature of the cell, an energy barrier of $1280 \pm 46 \mathrm{~cm}^{-1}$ was determined, close to the endoergicity of $1624 \mathrm{~cm}^{-1}(0.20 \mathrm{eV})$. With this excitation, no population was found in the $\mathrm{X}^{1} \Sigma^{+}$ $\left(v^{\prime \prime}=1\right)$ level.

The $\mathrm{Li}\left(3 \mathrm{~s}{ }^{2} \mathrm{~S}\right)+\mathrm{H}_{2}$ exoergic reaction was attempted with a two-step electronic excitation of the lithium atom, both under bulk and crossed-beam conditions. Unfortunately, no signal was detected, the cross-section being certainly lower than $0.05 \AA^{2}$ (Chen et al. 2001, R. Vetter 2002, unpublished results).

The system is better known on theoretical grounds (Rossi and Pascale 1985, Martinez 1997, Gianturco et al. 1998, 2001). The most recent study about PESs was achieved by ab initio methods in which extensive configuration interactions were carried out for multiple geometries of approach (Lee et al. 1999). They showed that the $\mathrm{Li}\left(2 \mathrm{p}^{2} \mathrm{P}\right)+\mathrm{H}_{2}$ channel with enough energy to overcome the residual endoergicity does lead to $\mathrm{LiH}$ formation through stable bent $\mathrm{Li}-\mathrm{H}_{2}$ intermediates, whereas the

$\dagger$ The endoergicity of an $\mathrm{A}+\mathrm{BC} \rightarrow \mathrm{AB}+\mathrm{C}$ reaction in its ground state is defined as the difference between the dissociation energies of $\mathrm{BC}$ and $\mathrm{AB}$ molecules assumed to be in their electronic-vibrational-rotational ground states. 

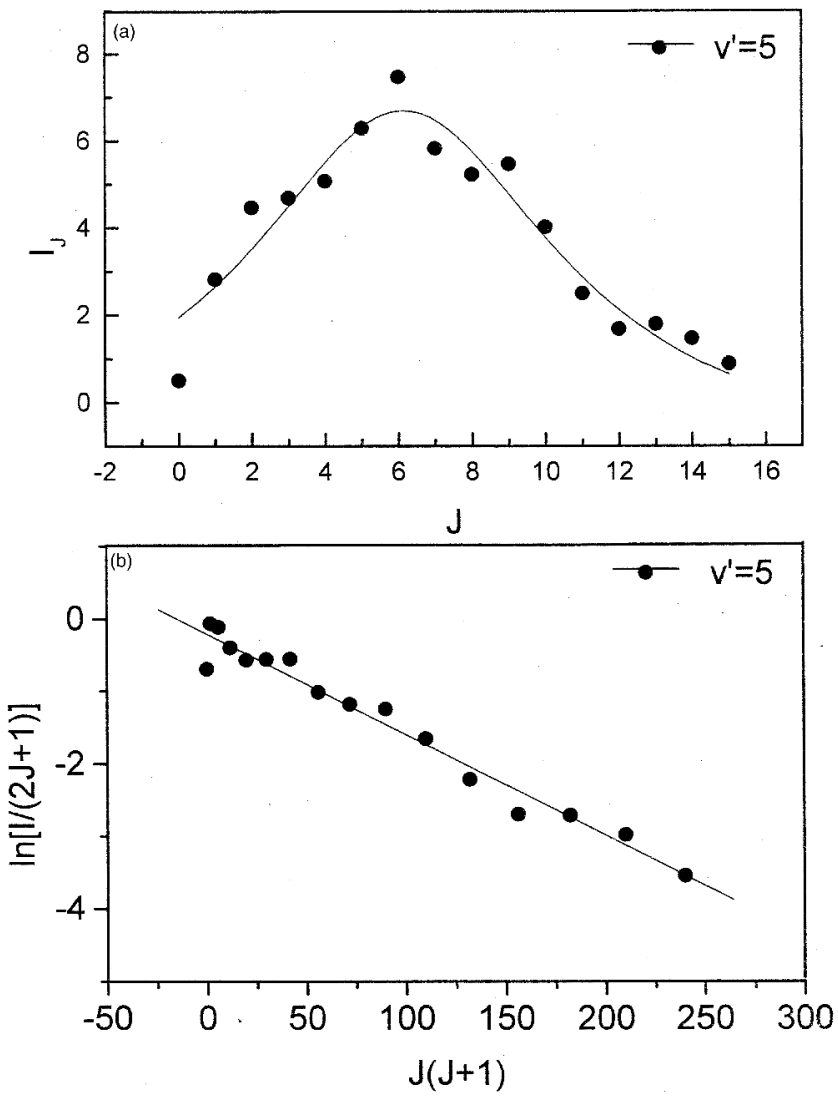

Figure 1. $\mathrm{Li}\left(2 \mathrm{p}^{2} \mathrm{P}\right)+\mathrm{H}_{2} \rightarrow \mathrm{LiH}+\mathrm{H}$ reaction. (a) The rotational state distribution of $\mathrm{LiH}$ $(v=0)$ determined from the absorption spectrum of the A-X $(5,0)$ band. $(b)$ A plot of $\ln [I /(2 J+1)]$ as a function of $J(J+1)$ in which $I$ denotes the peak area of the rotational line. The slope of the best-fit line leads to the Boltzmann rotational temperature. (Adapted from Chen et al. (2001).)

$\operatorname{Li}\left(3 \mathrm{~s}{ }^{2} \mathrm{~S}\right)+\mathrm{H}_{2}$ channel does not because it is efficiently quenched by the first. Another interesting feature is that the charge transfer from the alkali atom to the molecule would not take place abruptly but rather progressively as the distance between the metal and the dimer decreases. This would imply that the harpooning model does not hold stricto senso (Jeung et al. 2001).

The $\mathrm{Li}\left(2 \mathrm{p}^{2} \mathrm{P}\right)+\mathrm{H}_{2}$ reaction should occur via a side-on attack along the $2 \mathrm{~A}^{\prime}-1 \mathrm{~A}^{\prime}$ non-adiabatic transition (figure 2), the rotational state distribution of $\mathrm{LiH}$ depending on the anisotropy of the $1 \mathrm{~A}^{\prime}$ surface. The $\mathrm{Li}-\mathrm{H}_{2}$ intermediate complex has a bent geometry, with a $\mathrm{LiH}$ bond length of $1.6 \AA$, close to the equilibrium distance of $\mathrm{LiH}$ molecules (Lee et al. 1999). Thus, if one hydrogen atom departs impulsively from the complex, the impulse force should cause the available energy conversion into vibration to be relatively small. This consideration, other than the small endoergicity, may explain the lack of population in $v^{\prime \prime}=1$.

In addition, as the $\mathrm{Li}\left(3 \mathrm{~s}^{2} \mathrm{~S}\right)$ state is excited, the $4 \mathrm{~A}^{\prime}$ surface easily couples to a $3 \mathrm{~A}^{\prime}$ repulsive surface in a near- $\mathrm{C}_{2 \mathrm{v}}$ symmetry of approach. The repulsion exerting over the collision complex makes the moiety break up into the products $\mathrm{Li}\left(2 \mathrm{p}^{2} \mathrm{P}_{\mathrm{J}}\right)$ and $\mathrm{H}_{2}$ 


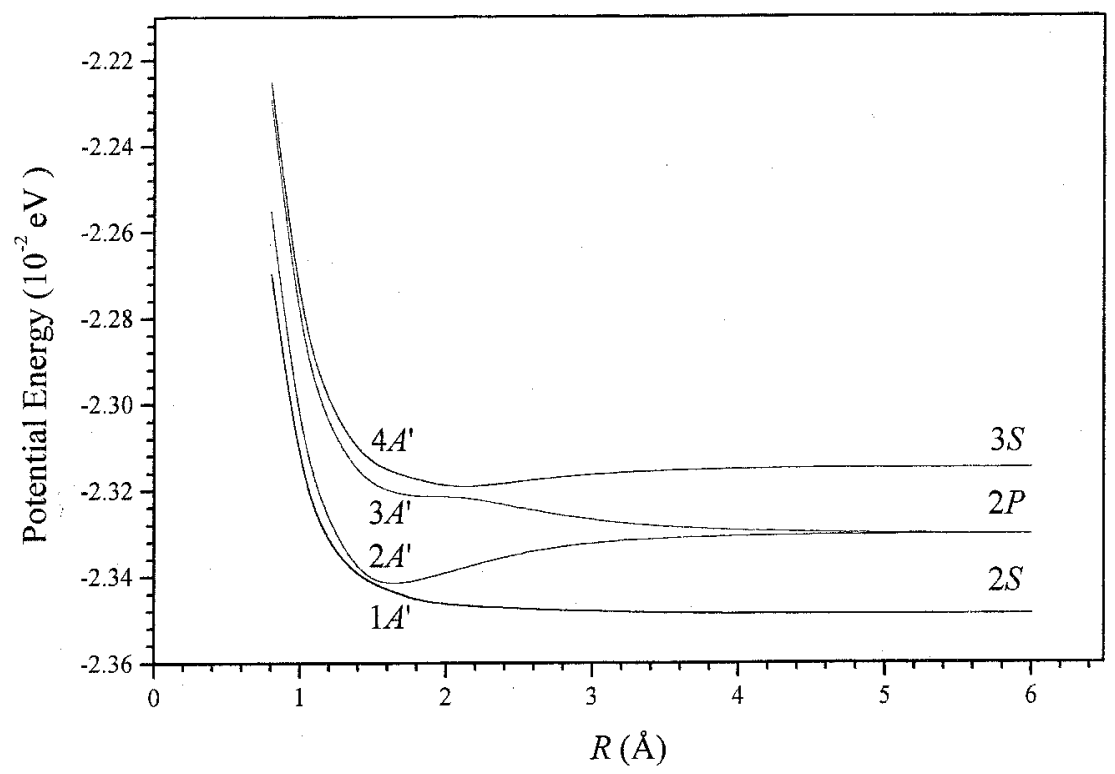

Figure 2. $\mathrm{Li}+\mathrm{H}_{2} \rightarrow \mathrm{LiH}+\mathrm{H}$ reaction. The $1 \mathrm{~A}^{\prime}$, the $2 \mathrm{~A}^{\prime}$, the $3 \mathrm{~A}^{\prime}$ and the $4 \mathrm{~A}^{\prime}$ PESs calculated in a near- $C_{2 \mathrm{v}}$ symmetry $\left(\right.$ angle, $89^{\circ}$ ). They correlate with the $2 \mathrm{~s}{ }^{2} \mathrm{~S}, 2 \mathrm{p}{ }^{2} \mathrm{P}$ and $3 \mathrm{~s}{ }^{2} \mathrm{~S}$ states, respectively of lithium. The $\mathrm{H}_{2}$ bond length is stretched to $0.9 \AA$. (Adapted from Chen et al. (2001).)

along the $3 \mathrm{~A}^{\prime}$ surface, the probability for further surface hopping from the $3 \mathrm{~A}^{\prime}$ surface on to the $2 \mathrm{~A}^{\prime}$ reactive surface being negligible since their energies are far too different to yield significant coupling. The $\mathrm{Li}\left(3 \mathrm{p}{ }^{2} \mathrm{P}\right)$ state is expected to behave similarly. The forbidden reactions found for these highly excited states of lithium imply that the harpooning mechanism is not dominant.

It is worthwhile to mention that other experimental techniques have led to noticeable improvements in the knowledge of the reactive system, the rate of $\mathrm{LiH}$ formation by electronic excitation of lithium atoms in electric discharges for example (Skenderovic et al. 2000). Also interesting is the reverse reaction, $\mathrm{LiH}+\mathrm{H} \rightarrow \mathrm{Li}+\mathrm{H}_{2}$, with release of lithium in $\mathrm{Li}\left(2 \mathrm{p}{ }^{2} \mathrm{P}\right)$ and $\mathrm{Li}\left(2 \mathrm{~s}{ }^{2} \mathrm{~S}\right)$ states. This reaction might be at the origin of the lack of $\mathrm{LiH}$ molecules found in the Universe light background at $3.2 \mathrm{~K}$ (Stancil et al. 1996).

\section{2. $\mathrm{Na}+\mathrm{H}_{2}$}

The $\mathrm{Na}+\mathrm{H}_{2} \rightarrow \mathrm{NaH}+\mathrm{H}$ reaction is endoergic by $2.60 \mathrm{eV}$ in its ground state. For this system, extensive pioneering calculations of ground-state and excited-state ab initio PESs were carried out long ago, showing the effective competition between reactive and non-reactive processes (Hertel et al. 1977, Hertel and Storl 1978, Hertel 1982). Later, the $\mathrm{Na}\left(3 \mathrm{p}^{2} \mathrm{P}\right)+\mathrm{H}_{2} \rightarrow \mathrm{NaH}+\mathrm{H}$ reaction, not allowed on an energy basis since it is endoergic by $0.5 \mathrm{eV}$, was nevertheless observed in a medium-pressure cell experiment using four-wave-mixing spectroscopic techniques of detection (Motzkus et al. 1998). The data analysis was performed in the framework of a two-step collision process involving vibrational excitation of hydrogen: 


$$
\begin{aligned}
& \mathrm{Na}(3 \mathrm{p})+\mathrm{H}_{2} \rightarrow \mathrm{Na}(3 \mathrm{~s})+\mathrm{H}_{2}\left(\mathrm{v}^{\prime \prime}=1,2,3\right), \\
& \mathrm{Na}(3 \mathrm{p})+\mathrm{H}_{2}\left(\mathrm{v}^{\prime \prime}=1,2,3\right) \rightarrow \mathrm{NaH}+\mathrm{H} .
\end{aligned}
$$

It led to the determination of the rate for $\mathrm{NaH}$ formation, other reaction schemes being ruled out from the detailed analysis of rate equations. The same technique of detection led to the determination of the time scale for $\mathrm{NaH}$ formation and to the measurement of their reactive and diffusive loss mechanisms in various gas atmospheres (Lehr et al. 1998).

The $\mathrm{Na}\left(4 \mathrm{p}^{2} \mathrm{P}\right)+\mathrm{H}_{2} \rightarrow \mathrm{NaH}+\mathrm{H}$ direct allowed reaction was studied in the bulk (Bililign and Kleiber 1992, Kleiber et al. 1993). A bimodal rotational state distribution was observed, which was interpreted assuming that the reaction stems from a side-on attack along an attractive surface that determines the microscopic branching late in the exit channel. The reaction proceeds mainly along the $3{ }^{2} \mathrm{~B}_{2}$ attractive surface in a side-on geometry of approach, evolves through a series of surface crossings and finally joins the $1{ }^{2} B_{2}$ reactive surface that correlates with the $\mathrm{Na}\left(3 \mathrm{p}{ }^{2} \mathrm{P}\right.$ ) state (figure 3) (Sevin and Chaquin 1985, Bililign and Kleiber 1992, Kleiber et al. 1993). This process gives rise to the major high rotational and the minor low rotational components of the state distribution. In a half-collision study with a far-wing excitation technique, orbital alignment was used to determine precisely the dynamics of the system (Bililign and Kleiber 1990, Bililign et al. 1992). As a result, a minor secondary mechanism was proposed, with an impulsive end-on attack along a repulsive surface. This process gives rise to the low-rotational component of the state distribution.

The two previous reactions were comparatively studied using three different nonlinear techniques of detection (Motzkus et al. 1997). It was shown that the differences observed in their vibrational state distributions were due to the different

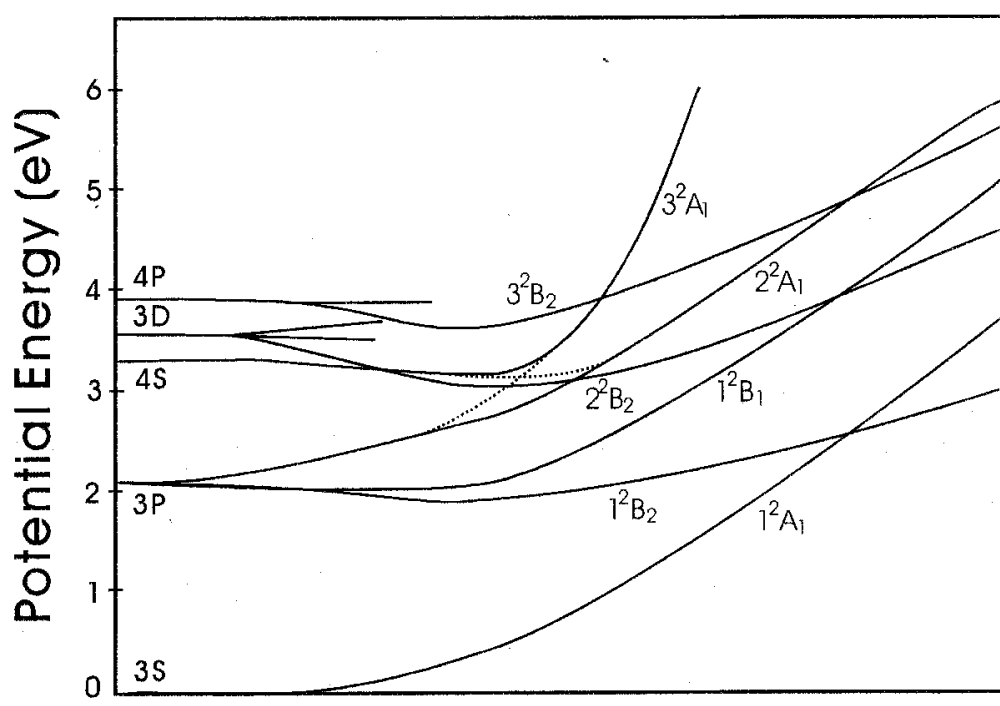

$R(\AA ̊)$

Figure 3. $\mathrm{Na}+\mathrm{H}_{2} \rightarrow \mathrm{NaH}+\mathrm{H}$ reaction. Scenario leading to the formation of $\mathrm{NaH}_{2}$ in a $C_{2 \mathrm{v}}$ symmetry. (Adapted from Sevin and Chaquin (1985).) 
shapes of the relevant PESs. The time scale for $\mathrm{NaH}$ formation from the $\mathrm{Na}\left(4 \mathrm{p}{ }^{2} \mathrm{P}\right)$ state was measured, confirming that the reaction is direct.

\section{3. $\mathrm{K}+\mathrm{H}_{2}$}

The $\mathrm{K}+\mathrm{H}_{2} \rightarrow \mathrm{KH}+\mathrm{H}$ reaction is endoergic by $2.62 \mathrm{eV}$ in its ground state. Systematic studies were performed by pump-probe techniques in low-pressure heatpipe oven experiments, for various excitations of the atom, namely $6 \mathrm{~s}{ }^{2} \mathrm{~S}, 7 \mathrm{~s}{ }^{2} \mathrm{~S}$, $5 p{ }^{2} \mathrm{P}, 6 \mathrm{p}{ }^{2} \mathrm{P}, 7 \mathrm{p}{ }^{2} \mathrm{P}, 4 \mathrm{~d}{ }^{2} \mathrm{D}$ and $5 \mathrm{~d}{ }^{2} \mathrm{D}$ (Liu and Lin 1996, 1997). They led to the determination of rovibrational state distributions together with those for translational energy (figure 4). The energy partitioning into translation, vibration and rotation, $-70 \%, 26 \%$ and $4 \%$ respectively, indicates that a harpooning-like mechanism dominates in these reactions via a collinear abstraction process. The
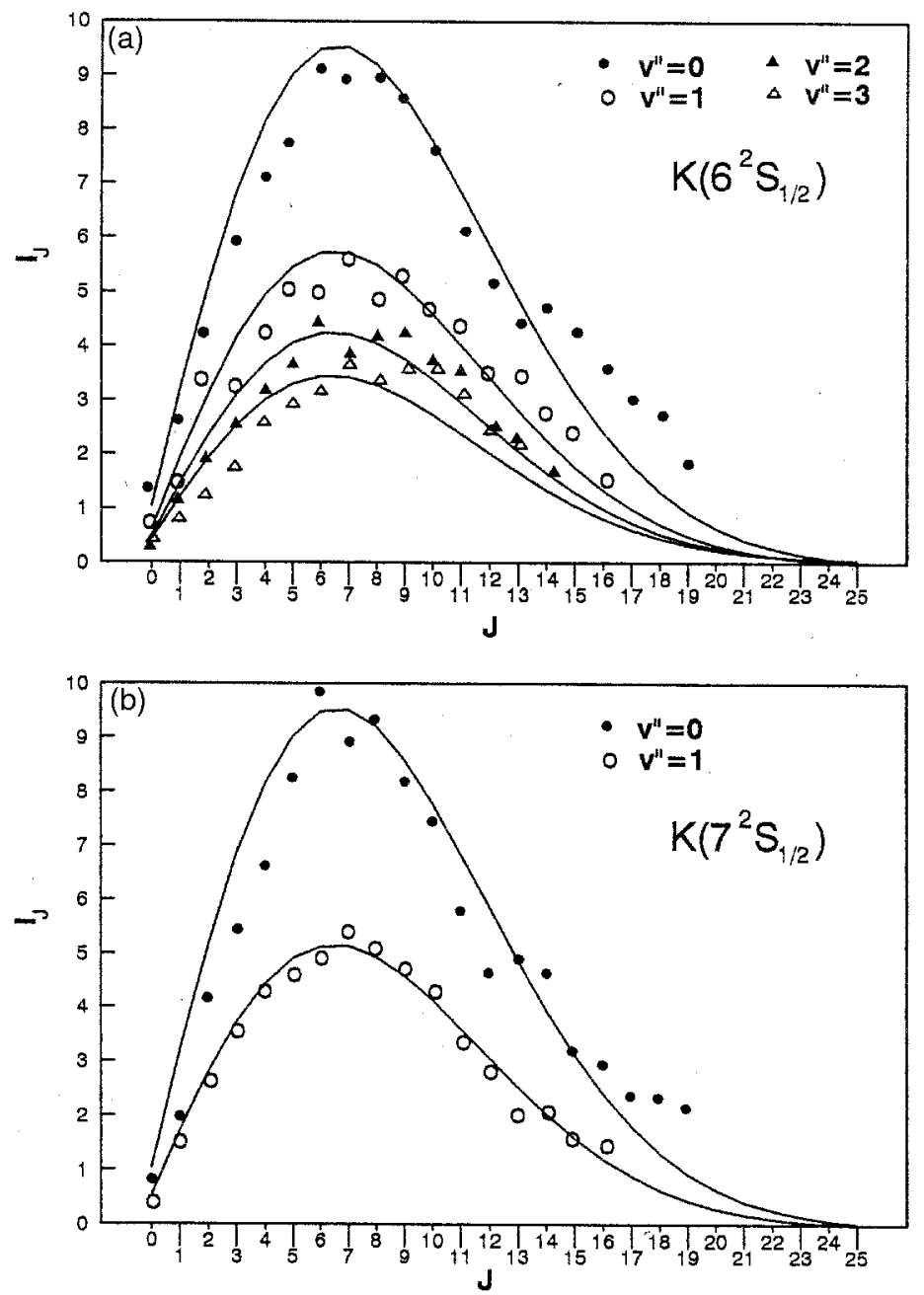

Figure 4. $\mathrm{K}+\mathrm{H}_{2} \rightarrow \mathrm{KH}+\mathrm{H}$ reaction. Rotational state distributions of $\mathrm{KH}\left(v^{\prime \prime}=0,1,2,3\right)$ and $\mathrm{KH}\left(v^{\prime \prime}=0,1\right)$ in $(a)$ the $\mathrm{K}\left(6 \mathrm{~s}^{2} \mathrm{~S}\right)+\mathrm{H}_{2}$ and $(b)$ the $\mathrm{K}\left(7 \mathrm{~s}{ }^{2} \mathrm{~S}\right)+\mathrm{H}_{2}$ reactions. Each solid curve represents the best least-squares thermal fit. (Adapted from Liu and Lin (1997).) 
energy release into translation is due to the instability and rupture of the $\mathrm{H}_{2}^{-}$bond that is strongly affected by the attraction between $\mathrm{K}^{+}$and $\mathrm{H}^{-}$, contrary to the predictions based on the DIPR model. This might be due to the negative electron affinity of $\mathrm{H}_{2}$ that induces a short distance for the electron jump, so that the $\mathrm{H}_{2}^{-}$ dissociation is not free from the influence of the atom.

Analysis of the data shows that the energy release into vibration increases with increasing term energy of the atomic state that is excited, and thus with the increasing elongation of the electron jump distance. To clarify this point, a harmonic oscillator model was successfully used to relate the energy disposal to the displacement from the KH equilibrium position (Liu and Lin 1997). It was surprising to find that, within experimental accuracy, the total energy release into rotation is almost irrespective of the various ${ }^{2} \mathrm{~S}$ or ${ }^{2} \mathrm{P}$ states of potassium, the fraction of rotational energy partitioning being, by far, much smaller than that expected from a prior distribution, two sevenths only. A collinear geometry of approach, with a weak rotational torque, is thus strongly suggested for the $\mathrm{K}\left(n \mathrm{~s}{ }^{2} \mathrm{~S}\right)$ and $\mathrm{K}\left(n \mathrm{p}{ }^{2} \mathrm{P}\right)$ reactions with $\mathrm{H}_{2}$.

The previous systematic results on electronic excitation indicate that the reactivity of potassium excited states is in the order $\mathrm{S}>\mathrm{P}>\mathrm{D}$, in agreement with the electronic correlations between reagents and products. In the harpooning mechanism, a linear $\mathrm{K}^{+} \mathrm{H}^{-}-\mathrm{H}$ ion pair intermediate may symmetrically correlate with $\mathrm{K}\left({ }^{2} \mathrm{~S},{ }^{2} \mathrm{P},{ }^{2} \mathrm{D}\right)$ and $\mathrm{H}_{2}\left({ }^{1} \Sigma_{\mathrm{g}}^{+}\right)$reagent states from one side and with $\left.\mathrm{KH}^{(}{ }^{1} \Sigma^{+}\right)$and $\mathrm{H}\left({ }^{2} \mathrm{~S}\right)$ product states through the ${ }^{1} \Sigma^{+}$symmetry from the other side. In a $C \infty_{\mathrm{V}}$ symmetry, the fractions for effective transitions between covalent and ionic surfaces correspond to one, one third, and one fifth for ${ }^{2} \mathrm{~S},{ }^{2} \mathrm{P}$ and ${ }^{2} \mathrm{D}$ orbitals respectively. This indicates that the key parameter that drives the reaction is the electronic orbital symmetry, and not the potential energy above the reaction threshold. On the contrary, Weiss et al. (1986) and Mestdagh et al. (1987) demonstrated in a crossed-beam experiment that the $\mathrm{Na}+\mathrm{O}_{2} \rightarrow \mathrm{NaO}+\mathrm{O}$ reaction is initiated from the $4 \mathrm{~d}^{2} \mathrm{D}$ state only, and not from the $3 \mathrm{p}^{2} \mathrm{P}$ or $5 \mathrm{~s}{ }^{2} \mathrm{~S}$ states. It was suggested, that for this last reaction, the $\mathrm{Na}^{+} \mathrm{O}_{2}^{-}\left(\mathrm{A}^{2} \Pi_{\mathrm{u}}\right)$ ion-pair intermediate should be a likely pathway connecting reagents to products.

The activation energies for the reactions of $\mathrm{K}\left(6 \mathrm{~s}{ }^{2} \mathrm{~S}\right), \mathrm{K}\left(7 \mathrm{~s}{ }^{2} \mathrm{~S}\right)$ and $\mathrm{K}\left(8 \mathrm{~s}{ }^{2} \mathrm{~S}\right)$ with hydrogen were determined by varying the temperature of the heat-pipe oven (Hsiao et al. 2000). They were estimated to be $5.38 \pm 0.33,4.39 \pm 0.16$ and $3.23 \pm 0.19 \mathrm{~kJ} \mathrm{~mol}^{-1}$ respectively, in rough agreement with the heights of the potential barriers predicted in a linear geometry of approach (Rossi and Pascale 1985). Such barriers are encountered before the electron transfer occurs and they are small enough $((1-2) k T)$ to let the harpooning mechanism happen. In a different experiment over the $\mathrm{K}\left(5 \mathrm{p}{ }^{2} \mathrm{P}\right)+\mathrm{H}_{2}$ reaction in which light scattering in the far wing of the atomic resonance line was detected, a strong blue-wing-red-wing asymmetry was observed in the reactive-to-non-reactive branching ratio (Fan et al. 1999, reference 23e). It led to the conclusion that the reactive system overcomes an activation barrier of $4.18 \pm 1.19 \mathrm{~kJ} \mathrm{~mol}^{-1}$ in a ${ }^{2} \Sigma^{+}$-like orbital alignment, in agreement with an electron jump mechanism through formation of a $\mathrm{K}^{+} \mathrm{H}^{-}-\mathrm{H}$ ion pair intermediate.

\section{4. $\mathrm{Rb}+\mathrm{H}_{2}$}

$\mathrm{The} \mathrm{Rb}+\mathrm{H}_{2} \rightarrow \mathrm{RbH}+\mathrm{H}$ reaction is endoergic by $2.73 \mathrm{eV}$ in its ground state. It has been far less studied than the other alkali-hydrogen reactions since only one 
experiment has been performed up to now, by pump-probe techniques in lowpressure heat-pipe ovens (Fan et al. 1999). The excitation of rubidium atoms to 5d ${ }^{2} \mathrm{D}_{3 / 2}, 5 \mathrm{~d}^{2} \mathrm{D}_{5 / 2}$ and $7 \mathrm{~s}^{2} \mathrm{~S}_{1 / 2}$ levels led to the determination of rotational distributions in the $v^{\prime \prime}=0,1$ and 2 ground state levels, with low rotational and high vibrational temperatures (figure 5). The measured relative reactivity is in the order $7 \mathrm{~s}^{2} \mathrm{~S}_{1 / 2}>5 \mathrm{~d}$ ${ }^{2} \mathrm{D}_{3 / 2}>5 \mathrm{~d}^{2} \mathrm{D}_{5 / 2}$; it shows the effect of fine structure upon reactivity. Most of the available energy $(80 \%)$ is channelled into translation and little into rotation. These results support the assumption of a harpooning mechanism in a collinear geometry of approach.

It is interesting to note that the collinear geometry of approach may lead not only to the chemical reaction but also to an effective electronic-to-vibrational energy
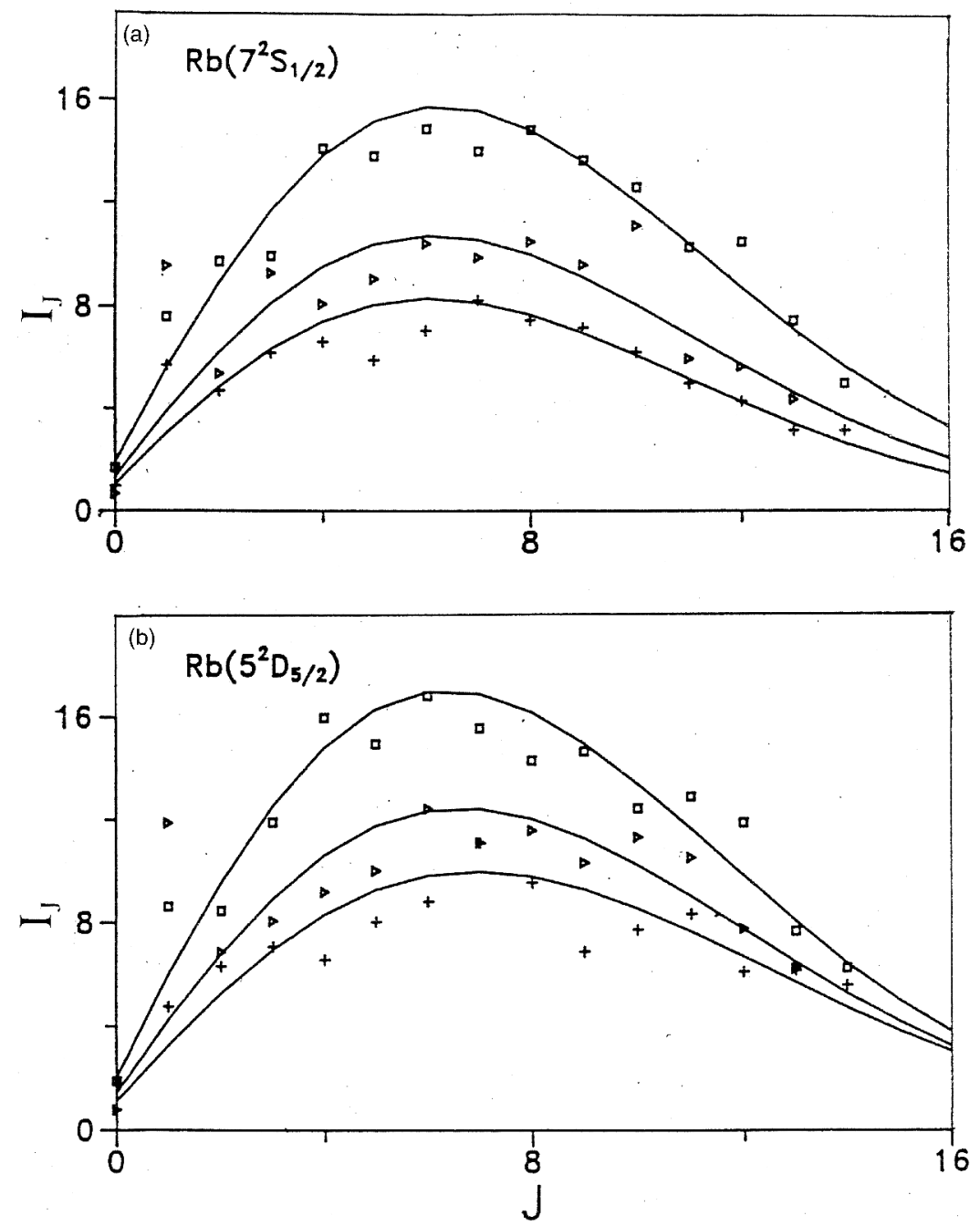

Figure 5. $\mathrm{Rb}+\mathrm{H}_{2} \rightarrow \mathrm{RbH}+\mathrm{H}$ reaction. Rotational state distributions of $\mathrm{RbH}\left(v^{\prime \prime}\right)$ in $(a)$ $\mathrm{Rb}\left(7 \mathrm{~s}^{2} \mathrm{~S}_{1 / 2}\right)+\mathrm{H}_{2}$ and $(b)$ the $\mathrm{Rb}\left(5 \mathrm{~d}{ }^{2} \mathrm{D}_{5 / 2}\right)+\mathrm{H}_{2}$ reactions: ( $\left.\square\right)$, distributions in $v^{\prime \prime}=0 ;(\triangleright)$, distributions for $v^{\prime \prime}=1 ;(+)$, distributions for $v^{\prime \prime}=2$. Each solid curve represents the best least-squares thermal fit. (Adapted from Fan et al. (1999).) 
transfer (Chen et al. 1997). Using CARS techniques to probe the relative vibrational populations of hydrogen induced by collisions with $\mathrm{Rb}\left(5 \mathrm{p}{ }^{2} \mathrm{P}\right)$ atoms, it was shown that $63 \%$ appear in $v^{\prime \prime}=2$ and $37 \%$ in $v^{\prime \prime}=1$, the efficiency of the $E-V$ energy transfer being 0.48 . These results are consistent with predictions based on the impulsive model.

\section{5. $\mathrm{Cs}+\mathrm{H}_{2}$}

The $\mathrm{Cs}+\mathrm{H}_{2} \rightarrow \mathrm{CsH}+\mathrm{H}$ reaction is endoergic by $2.7 \mathrm{eV}$ in its ground state. The $\mathrm{Cs}\left(7 \mathrm{p}^{2} \mathrm{P}\right)+\mathrm{H}_{2}$ photochemical reaction was first evidenced by the well-known 'laser snow' experiment in which electronic excitation of caesium atoms to the $7 \mathrm{p}^{2} \mathrm{P}$ state in a gas cell heated at $600 \mathrm{~K}$ gave rise to formation of $\mathrm{CsH}$ molecules followed by condensation into micron-size particles (Tam et al. 1975, Picqué et al. 1980). This photochemical reaction is particularly striking since it starts from the eleventh potential surface above ground state to yield $\mathrm{CsH}$ products in the $\mathrm{X}^{1} \Sigma^{+}$ground state, without energy loss (Crépin et al. 1984); indeed, the Cs $\left(7 \mathrm{p}^{2} \mathrm{P}_{1 / 2}\right)+\mathrm{H}_{2}$ reaction is strictly isoergic and the $\mathrm{Cs}\left(7 \mathrm{p}{ }^{2} \mathrm{P}_{3 / 2}\right)+\mathrm{H}_{2}$ reaction is exoergic by $0.02 \mathrm{eV}$. They were studied in a cell experiment (Sayer et al. 1981, Visticot et al. 1983) and later in a crossed-beam experiment that revealed the detailed dynamics of the reaction (Crépin et al. 1984, Rahmat et al. 1986, 1987, Gadéa et al. 1988, L'Hermite et al. 1990a, b, 1991, Cavero et al. 1999). In parallel, ab initio potential surfaces were calculated for this reaction in the framework of diabatic approximations (Gadéa et al. 1986).

The crossed-beam experiment included an effusive beam of caesium atoms and a supersonic beam of hydrogen, a first continuous-wave tuneable laser beam in the blue to excite caesium atoms up to a well-defined fine or hyperfine structure level of the $7 \mathrm{p}^{2} \mathrm{P}$ state and a second continuous-wave tuneable laser beam in the green to detect and to characterize $\mathrm{CsH}$ products by laser-induced fluorescence. The rotationally resolved excitation functions were determined by varying the temperature of the hydrogen beam. They led to the conclusion that the reaction proceeds without substantial potential barrier in the entrance valley of the reaction path, in any case not higher than $0.015 \mathrm{eV}$, in agreement with the $a b$ initio calculations. The rotational state distributions in the $\mathrm{X}^{1} \Sigma^{+}\left(v^{\prime \prime}=0\right)$ level of $\mathrm{CsH}$ were determined at two collision energies: 0.09 and $0.045 \mathrm{eV} . \dagger$ They are close to statistical prior distributions, with the same weak 'surprisal' (Levine and Bernstein 1987), most of the available energy being released into translation $(70 \%)$ and little into rotation $(30 \%)$ for both collision energies. The rotationally resolved differential angular cross-sections were studied at various collision energies by analysing the shapes of CsH absorption profiles ('Doppler technique'). These profiles were fairly well fitted assuming a 'forward' peaking of products at small angles superimposed to a flat background at all angles in the centre-of-mass framework. At the limit of experimental accuracy, it was shown that this forward peaking narrows on increasing the collision energy (figure 6).

The shapes of rotational and angular distributions suggest that the reaction might proceed through two different mechanisms: a direct mechanism leading to forward scattering and an indirect mechanism leading to isotropic scattering. The coexistence of these two mechanisms was confirmed by QCT calculations performed

$\dagger$ For this reaction, the magnitude of the reagent kinetic energy was enough to populate the $\mathrm{X}^{1} \Sigma^{+}\left(v^{\prime \prime}=0\right)$ level of $\mathrm{CsH}$ but not $v^{\prime \prime}=1$. 

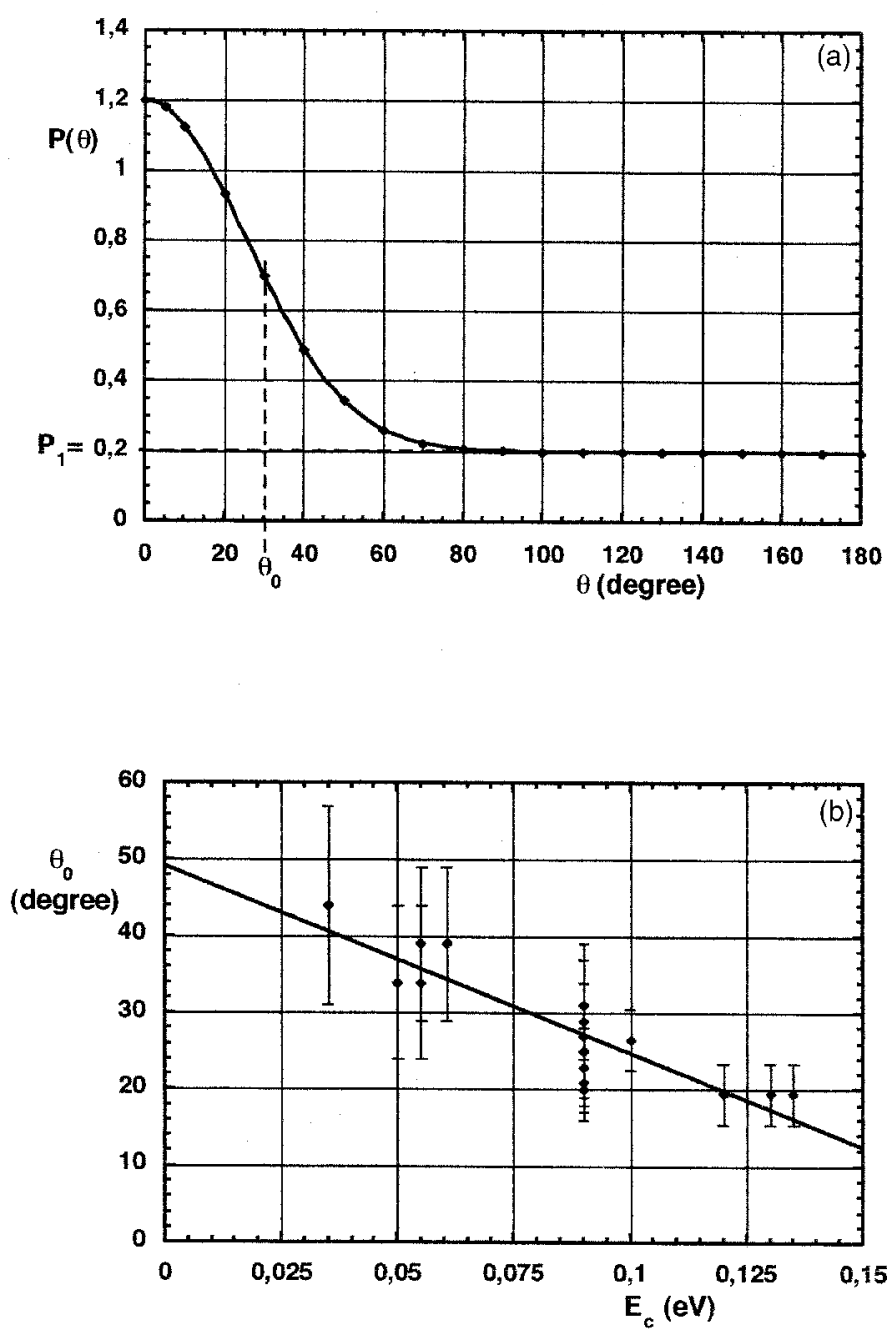

Figure 6. $\mathrm{Cs}\left(7 \mathrm{p}^{2} \mathrm{P}\right)+\mathrm{H}_{2} \rightarrow \mathrm{CsH}+\mathrm{H}$ reaction. The probability $\mathrm{P}(\theta)$ for angular scattering in the centre-of-mass framework has been obtained by fitting $\mathrm{CsH}$ absorption profiles with $P(\theta)=P_{1}+P_{2} \exp \left\{-\left[(\ln 2)\left(\theta^{2} / \theta_{0}^{2}\right)\right]\right\}$. (a) $P(\theta)$ versus $\theta$, with an angle $\theta_{0}=30^{\circ}$ and $P_{2} / P_{1}=5$ for a reagent kinetic energy $E_{\mathrm{c}}=0.09 \mathrm{eV}$. (b) The variation in the scattering angle $\theta_{0}$ versus reagent kinetic energy, with a marked narrowing in the kinetic energy range. In this energy range, $P_{2} / P_{1}$ is constant to experimental accuracy. (Adapted from L'Hermite et al. (1990a, b).)

in the framework of a harpooning model, assuming that direct and indirect collisions are likely to occur once the electron jump has occurred (L'Hermite 1992). Direct collisions were associated with the usual harpooning mechanism, whereas indirect collisions were related to the formation of an osculating complex due to charge migrations between the two hydrogen atoms, this complex dissociating at all angles in the centre-of-mass framework.

Relative intensity measurements revealed that the cross-section for the Cs $(7 \mathrm{p}$ $\left.{ }^{2} \mathrm{P}_{1 / 2}\right)+H_{2}$ reaction at threshold is substantially larger than that for the $\operatorname{Cs}\left(7 \mathrm{p}{ }^{2} \mathrm{P}_{3 / 2}\right)$ exoergic reaction. This was interpreted by hemiquantal dynamics calculations 
showing that the key parameter that determines the cross-section is not the potential energy but the electronic symmetry of the system, at the crossing distance between the $\mathrm{Cs}\left(7 \mathrm{p}{ }^{2} \mathrm{P}\right)+\mathrm{H}_{2}$ neutral entrance valley and the $\mathrm{Cs}^{+}-\mathrm{H}_{2}^{-}$ionic intermediate (Gadéa and Durup 1987). This result was confirmed by measuring the cross-sections relative to the various hyperfine structure levels of the $7 \mathrm{p}^{2} \mathrm{P}$ state (Gadéa et al. 1988). Absolute cross-section measurements were also carried out, although with a poor accuracy; they led to total cross-sections smaller than $1 \AA^{2}$ (Cavero et al. 1999).

At first sight, the harpooning mechanism seems dominant in this reaction. On the contrary, the small total cross-sections and the shapes of rotational and angular distributions suggest that the reaction also proceeds through a stable $\mathrm{Cs}-\mathrm{H}_{2}$ intermediate, in agreement with QCT calculations. The weakness of vibrational and rotational product excitations may be attributed to the fact that the electron transfer occurs at short internuclear distances, $3.5 \AA$ typically, close to the $\mathrm{CsH}$ equilibrium bond length (Gadéa et al. 1986).

Higher excitations to $\operatorname{Cs}\left(6 d{ }^{2} D\right)$ and $\operatorname{Cs}\left(8 s{ }^{2} S\right)$ were tried with the previous crossed-beam experiment, showing smaller and nil reactive cross-sections respectively (Cavero et al. 1999). In a different experiment with $\operatorname{Cs}\left(8 \mathrm{p}{ }^{2} \mathrm{P}\right)$ and $\operatorname{Cs}\left(9 \mathrm{p}{ }^{2} \mathrm{P}\right)$ excitations (Huang et al. 1996), it was found that the fine structure of the atom plays no role over reactivity and that most of the available energy $(90 \%)$ is released into translation, $\mathrm{CsH}$ products being found in the $v^{\prime \prime}=0$ and $v^{\prime \prime}=1$ levels only. This was interpreted by assuming that the reaction proceeds via a collinear abstraction, with an electron transfer occurring at short internuclear distances.

\section{Alkali-hydrogen reactions: the general trends}

In spite of their common characteristics, alkali-hydrogen photochemical reactions exhibit apparently different behaviours that are not explained by usual collision models. As the endoergicity is compensated by the electronic excitation of the alkali atom, the system becomes isoergic or slightly exoergic with respect to the formation of products in their electronic ground states. The reagent system jumps from the excited $\mathrm{A}-\mathrm{H}_{2}$ entrance valley on to the ground state $\mathrm{AH}+\mathrm{H}$ exit valley, in which the diatomic product presents a pronounced ionic character at short distances. Accordingly, an electron transfer necessarily occurs during the collision process, from the alkali to the hydrogen molecule, to form an $\mathrm{A}^{+} \mathrm{H}^{-}-\mathrm{H}$ ionic intermediate that dissociates into $\mathrm{A}^{+} \mathrm{H}^{-}+\mathrm{H}$. This non-adiabatic process competes with quenching non-reactive processes that are associated with the other underlying potential surfaces. As a consequence, the reaction cross-section is generally small, of the order of square ångstroms or less. By comparison, exoergic alkali-halogen reactions proceed over adiabatic ground-state PESs and the electron jump takes place at the crossing distance between the neutral entrance valley and the ionic intermediate. As this electron jump has a strong probability of occurrence and the crossing distance is long, the cross-section is large, $100 \AA^{2}$ or more.

This simple argument holds, suggesting that, contrary to previous assumptions, these two types of reaction do not imply the same type of mechanism, the question being to determine how and when the electron transfer does occur in alkalihydrogen reactions. A first answer was provided by ab initio calculations of PESs by Jeung et al. (2001) on the $\mathrm{Li}-\mathrm{H}_{2}, \mathrm{Na}-\mathrm{H}_{2}$ and $\mathrm{K}-\mathrm{H}_{2}$ systems. They have shown that the electron population is transferred progressively from the metal to the diatomic as the internuclear distance decreases, with a constant short distance 
between the two hydrogen atoms. This is due to the fact that the equilibrium distances are nearly the same for $\mathrm{HH}$ and $\mathrm{HH}^{-}$. Since the A-HH complex is compact, it needs both a high collision energy and a long lifetime to produce internal vibrations with enough amplitude to expel a hydrogen atom. Furthermore, no argument ensures that the electron charge stays on the same hydrogen atom, as was suggested by QCT calculations over the $\mathrm{Cs}+\mathrm{H}_{2}$ reaction (L'Hermite 1992). The same kind of progressive transfer occurs in the $\mathrm{Ca}+\mathrm{H}_{2}$ reaction (Kim et al. 2002). By comparison, for alkali-halogen reactions, the electron jump occurs abruptly at long distances, $10 \AA$ for the $\mathrm{Li}+\mathrm{F}_{2}$ reaction. The distance between the two halogens increases noticeably during the collision approach, the equilibrium distance being much longer in $\mathrm{XX}^{-}$than in XX. This favours the departure of an $\mathrm{X}$ atom and the high vibrational excitation of the MX product that is allowed by the high exoergicity of the reaction.

In these mechanisms, the electronic symmetry of the reagent-product system plays a major role. In a definite geometry of approach, the reagent covalent states have well-defined orbital components that have to match with those of the ionic intermediate. In other words, the probability of transfer is a maximum when the incoming covalent wavefunction and the ionic wavefunction are able to couple efficiently. Consider for example the reactions from the excited states of potassium, for which the relative reaction cross-sections follow the trend $\mathrm{S}>\mathrm{P}>\mathrm{D}$ under similar energy conditions (Liu and Lin 1996). Indeed, in a collinear geometry of approach, the fractions of effective transitions between covalent and ionic surfaces should correspond to one, one-third and one-fifth for S, P and D orbitals respectively, in accordance with the extent of the wavefunction penetration into the nuclear core of the atom. As the electron transfer does depend on the wave function overlap, the probability for hopping on to the ionic surface follows the same trend. This state selectivity was also observed in the $\mathrm{Rb}+\mathrm{H}_{2}$ and $\mathrm{Cs}+\mathrm{H}_{2}$ reactions (Cavero et al. 1999, Fan et al. 1999).

At a more specific level, the fine structure of the atom may also play a role since it influences the composition of wavefunctions. In the $\mathrm{Cs}\left(7 \mathrm{p}{ }^{2} \mathrm{P}\right)+\mathrm{H}_{2}$ reaction for example, the Cs $\left(7 \mathrm{p}^{2} \mathrm{P}_{1 / 2}\right)$ level is four times more reactive than the $\mathrm{Cs}\left(7 \mathrm{p}^{2} \mathrm{P}_{3 / 2}\right)$ level in spite of its lower excitation energy (Gadéa and Durup 1987, L'Hermite et al. 1991). This result was explained by considering the electronic symmetry of the system: out of the ${ }^{2} \Pi$ and ${ }^{2} \Sigma$ covalent incoming states only the second states are able to induce transitions to the ionic intermediate of ${ }^{2} \Sigma$ symmetry. At infinite distances, the weights of ${ }^{2} \Sigma$ components are the same for the two channels associated with $\operatorname{Cs}\left(7 \mathrm{p}{ }^{2} \mathrm{P}_{1 / 2}\right)$ and $\operatorname{Cs}\left(7 \mathrm{p}{ }^{2} \mathrm{P}_{3 / 2}\right)$ but, owing to strong spin-orbit couplings in the entrance valley, the weight of the ${ }^{2} \Sigma$ component becomes larger for the $\operatorname{Cs}\left(7 \mathrm{p}^{2} \mathrm{P}_{1 / 2}\right)$ level than for the $\operatorname{Cs}\left(7 \mathrm{p}^{2} \mathrm{P}_{3 / 2}\right)$ level, at the crossing distance between the covalent entrance valley and the ionic intermediate. This interpretation was confirmed by measuring the cross-sections related to the various hyperfine structure levels of the $7 \mathrm{p}{ }^{2} \mathrm{P}$ state (caesium has a nuclear spin of $7 / 2$ ) versus the angle between the polarization axis of the laser beam that excites caesium atoms and the collision axis taken here as quantification axis (Gadea et al. 1988). By rotating the polarization axis, one changes the relative population inside a given set of magnetic hyperfine sublevels of the $7 \mathrm{p}^{2} \mathrm{P}_{1 / 2,3 / 2}$ levels, and in particular the population of the extreme sublevels. If these sublevels are more reactive than the others, that is their ${ }^{2} \Sigma$ weight is larger, a relative variation in the cross-section may occur. The predictions of this model were confirmed since, as expected for two $6{ }^{1} \mathrm{~S}_{0}-7 \mathrm{p}{ }^{2} \mathrm{P}_{3 / / 2}$ hyperfine 
transitions, large variations $(30 \%)$ in the cross-sections were observed between parallel and perpendicular orientations of the polarization. For the eight other hyperfine transitions, no significant variations were predicted and detected at experimental accuracy. A fine-structure effect was also observed in the $\mathrm{Rb}+\mathrm{H}_{2}$ reaction, with the same ordering for reactivity, $5 \mathrm{~d}^{2} \mathrm{D}_{3 / 2}>5 \mathrm{~d}^{2} \mathrm{D}_{5 / 2}$, and the same interpretation (Fan et al. 1999).

The magnitude of the excitation energy may also affect the mechanism. If an atom is pumped up to a highly excited state, ionization becomes easier and may enhance the rate for electron transfer. For instance, the $\mathrm{Ca}\left(4 \mathrm{~s}^{2}{ }^{1} \mathrm{~S}_{0}\right)+\mathrm{H}_{2} \mathrm{O}_{2}$ groundstate reaction proceeds via an insertion mechanism to yield a neutral long-lived complex that dissociates predominantly into $\mathrm{CaO}$ and $\mathrm{H}_{2} \mathrm{O}$ molecules, whereas the $\mathrm{Ca}\left(4 \mathrm{~s} 4 \mathrm{p}{ }^{3} \mathrm{P}_{j}\right)+\mathrm{H}_{2} \mathrm{O}_{2}$ reaction is dominated by an electron transfer to yield $\mathrm{CaOH}$ and $\mathrm{OH}$ products (Oberlander et al. 1991). The $\mathrm{Mg}\left(3 \mathrm{~s} 3 \mathrm{p}{ }^{1} \mathrm{P}_{1}\right)+\mathrm{H}_{2}$ reaction similarly proceeds via an insertion mechanism, whereas the $\mathrm{Mg}\left(3 \mathrm{~s} 3 \mathrm{~d}{ }^{1} \mathrm{D}_{2}\right)+\mathrm{H}_{2}$ reaction is believed to proceed via a harpooning-type process (Liu et al. 2000). Such a competition between insertion and harpooning mechanisms has also been found in other alkaline earth atoms reacting with $\mathrm{OH}$-containing molecules (De Pujo et al. 1993). Comparing the ionization potentials among the excited states of sodium, rubidium, potassium and caesium atoms studied so far indicates that the $\mathrm{Na}\left(4 \mathrm{p}{ }^{2} \mathrm{P}\right)$ atom is the most difficult to ionize. Indeed, for this reaction, the harpooning process is less competitive.

As shown above, the reaction mechanisms appear different from lithium to caesium. The $\mathrm{Li}\left(2 \mathrm{p}{ }^{2} \mathrm{P}\right)+\mathrm{H}_{2}$ and $\mathrm{Na}\left(4 \mathrm{p}^{2} \mathrm{P}\right)+\mathrm{H}_{2}$ reactions occur preferentially through a side-on attack towards the hydrogen molecule, leading to a unimodal rotational distribution for $\mathrm{LiH}$ and a bimodal rotational distribution for $\mathrm{NaH}$. In contrast, the $\mathrm{K}+\mathrm{H}_{2}, \mathrm{Rb}+\mathrm{H}_{2}$ and $\mathrm{Cs}+\mathrm{H}_{2}$ reactions occur along a collinear geometry of approach via an electron transfer. According to Huang et al. (1996), the atomic size of the alkali elements may be an important factor to determine the preference for the reaction pathways; lithium and sodium atoms with a relatively small size tend to insert into the $\mathrm{H}_{2}$ bond with the least repulsion, whereas potassium, rubidium and caesium atoms with a larger size may encounter a larger repulsion to insert between the two hydrogen atoms. For lithium and sodium, an efficient electron back donation from the metal atom to the antibonding $\sigma^{*}$ orbital of the hydrogen molecule is another important factor that facilitates the insertion mechanism since it may energetically stabilize the ${ }^{2} \mathrm{~B}_{2}\left(\mathrm{or}^{2} \mathrm{~A}^{\prime}\right)$ potential surface. The influence of atomic size was also observed in the $\mathrm{Mg}\left(3 \mathrm{~s} 3 \mathrm{p}{ }^{1} \mathrm{P}\right)+\mathrm{H}_{2}$ and $\mathrm{Ca}(4 \mathrm{~s} 4 \mathrm{p}$ $\left.{ }^{1} \mathrm{P}\right)+\mathrm{H}_{2}$ reactions; the former case is dominated by an insertion mechanism (Breckenridge and Umemoto 1984, Ou et al. 1998, Hung and Lin 2001) while the latter occurs with a collinear geometry of approach and leads to $\mathrm{CaH}$ products in low rotational but high vibrational states (Liu et al. 1999).

Since the electron affinity of the hydrogen molecule is negative, the distance for electron transfer might lead to significant impact on the chemical reaction. For the $\mathrm{K}+\mathrm{H}_{2}$ reaction, as the potassium atom ejects an electron towards the $\mathrm{H}_{2}$ molecule, a repulsive force is exerted on $\mathrm{H}_{2}{ }^{-}$which causes an abrupt dissociation, but the electron jump distance is not large enough to ignore the coupling between repulsion and attraction. The repulsion has to be considered between the hydrogen atom and $\mathrm{K}^{+} \mathrm{H}^{-}$as a whole, rather than considered between the hydrogen atom and $\mathrm{H}^{-}$. The resulting coupling between repulsive and attractive energies exhibits two major features: firstly, the increase in repulsion enhances the available energy partitioning 
into translation and, secondly, the fraction of repulsive energy that goes to vibration increases with increasing atomic masses. In contrast, for $\mathrm{K}+\mathrm{X}_{2}$ reactions, the halogen molecule has a strong electron-withdrawing character. Thus, repulsion and attraction may be considered to be sequential and separable and the repulsive energy acting on the $\mathrm{X}_{2}^{-}$bond rupture becomes independent of the electron jump distance.

By using this concept of mixed energy release, the comparison of product energy partitioning among the $\mathrm{K}+\mathrm{H}_{2}, \mathrm{Rb}+\mathrm{H}_{2}$ and $\mathrm{Cs}+\mathrm{H}_{2}$ reactions should be rationalized. Indeed, if this consideration interprets the fact that the fractions of vibrational energy in $\mathrm{KH}(26 \%), \mathrm{RbH}(17 \%)$ and $\mathrm{CsH}$ products $(10 \%)$ are much smaller than that observed in $\mathrm{KBr}$ products (greater than $60 \%$ ) resulting from the $\mathrm{K}+\mathrm{Br}_{2}$ reaction, it does not interpret the order for vibrational energy, $\mathrm{KH}>\mathrm{RbH}>\mathrm{CsH}$, which is observed. These contradictory findings imply that the electron jump distance should be reduced for $\mathrm{Cs}+\mathrm{H}_{2}$ and $\mathrm{Rb}+\mathrm{H}_{2}$ reactions, as suggested by Huang et al. (1996). The electron jump distance was estimated to be $3.5 \AA$ for the $\mathrm{Cs}+\mathrm{H}_{2}$ reaction (Gadéa et al. 1986).

In summary, the way in which the electron transfer occurs along the reaction path is the key factor that drives the alkali-hydrogen photochemical reaction, the amount of potential energy above the reaction threshold playing a minor role. Other parameters such as the size and the excitation energy of the alkali atom, the height of potential barriers also determine the reaction mechanism, side-on insertion for lithium and sodium, collinear abstraction for potassium, rubidium and caesium. For this last atom, a stable intermediate is likely to occur.

\section{Conclusions}

It thus appears that, if some general trends can be foreseen in the characteristics of alkali-hydrogen reactions, the situation demands more clarification. It necessarily requires knowledge of more detailed and more extensive experimental data over angular differential cross-sections to determine whether the reaction is direct or not, over fine-structure cross-sections to generalize the effect of electronic symmetry, and over excitation functions to determine the effect of potential barriers on the reaction mechanism, an effect that is not yet completely understood. In parallel, ab initio calculations of the relevant excited potential surfaces including spin-orbit effects are fundamentally important, as are three-dimensional dynamics calculations in which surface jumps are correctly treated.

With hydrogen molecules, one is not able to use the new powerful tools that are provided by the spatial orientation of molecular reagents developed by Loesch and Reincheid $(1990,1991)$ or by the STIRAP vibrational excitation technique developed by Gaubatz et al. (1989) and Schiemann et al. (1993). However, the product imaging technique developed by Suits et al. (1992) can probably be advantageous to study alkali-hydrogen reactions, with a sensitive detection of reaction products and a three-dimensional quantum state-specific characterization of angular scattering. With existing experimental techniques, it is possible to probe kinematic effects by using isotopic compounds and to study vectorial properties via the orbital alignment of atomic reagents, up to now the only way to gain information about the stereodynamics of these reactions. It is also possible to probe the dynamics of van der Waals complexes (the weakly bound $\mathrm{Li}-\mathrm{H}_{2}$ for instance) which, once excited, lead to reaction with fixed geometries (Jouvet and Soep 1984, Jouvet et al. 1989). 
The reverse reactions $\mathrm{AH}+\mathrm{H} \rightarrow \mathrm{A}+\mathrm{H}_{2}$ are certainly most interesting to study under crossed-beam conditions. They are necessarily highly exoergic, with two open channels.

(i) Reaction over the adiabatic ground state potential surface. It provides alkali atoms in their ground state and vibrationally excited hydrogen molecules, the excess energy being kinetic energy.

(ii) Reaction with one to several surface jumps (lithium to caesium) via nonadiabatic processes. It provides excited alkali atoms and vibrationally excited hydrogen molecules, the excess energy being kinetic energy.

The discrimination between these two channels can probably be achieved by using the usual tools of high-resolution spectroscopy. As already noted, the $\mathrm{LiH}+\mathrm{H}$ reaction might be at the origin of the lack of $\mathrm{LiH}$ molecules in the Universe light background (Stancil et al. 1996).

The collision intermediates are also most interesting to observe directly, to check the data provided by ab initio calculations, the widths and depths of intermediate wells for instance. The characterization of $\mathrm{A}^{+}-\mathrm{H}_{2}^{-}$ion pairs could confirm that the harpooning process takes place during the reactive collision. These experiments are difficult to achieve by studying the direct reactions. They are probably easier by studying the reverse reactions that do not need electronic excitation; hence we have a more sensitive optical detection of the reaction products, and the alkali atoms more precisely.

Other chemical trends have been observed in many fields of organic and inorganic chemistry, as well as in cluster physics. To our knowledge, this review about alkali-hydrogen photochemical reactions constitutes one of the rare attempts to generalize our understanding of non-adiabatic processes in reaction dynamics.

\section{Acknowledgements}

The authors are pleased to thank W. Y. Lee and J. J. Chen for preparing the figures for this article. They acknowledge the contributions of the National Science Council, Taiwan, and of the DRI, Centre National de la Recherche Scientifique, France, for travelling support. One of us (K.C.L.) is grateful for the financial support of the National Science Council, Taiwan, under contract NSC 90-2113-M001-032.

\section{References}

Becker, C. H., Casavecchia, P., Tiedemann, P. W., Valentini, J. J., and Lee, Y. T., 1980, J. chem. Phys., 73, 2833

Bergmann, K., 1988, Atomic and Molecular Beam Methods, I, edited by G. Scoles (Oxford University Press), p. 293.

Bernstein, R. B., 1982, Chemical Dynamics via Molecular Beams and Laser Techniques (Oxford University Press); 1986, Recent Advances in Molecular Reaction Dynamics, edited by R. Vetter and J. Vigué (Paris: Editions du CNRS), p. 51.

Bernstein, R. B. (editor), 1984, Atom-Molecule Collision Theory, A Guide for the Experimentalist (New York: Plenum).

Bernstein, R. B., and Rulis, A. M., 1973, Discuss. Faraday Soc., 55, 293.

Bililign, S., and Kleiber, P. D., 1990, Phys. Rev. A, 42, 6938; 1992, J. chem. Phys., 96, 213.

Bililign, S., Kleiber, P. D., Kearny, W. R., and Sando, K. M., 1992, J. chem. Phys., 96, 218. 
Blais, N. C., and Truhlar, D. G., 1983, Chem. Phys. Lett., 102, 120.

Breckenridge, W. H., and Umemoto, H., 1981, J. chem. Phys., 75, 4153; 1984, ibid, 80, 4168.

Brouard, M., Duxon, S. P., Enriquez, P. A., Sayos, R., and Simons, J. P., 1991, J. phys. Chem., 95, 8169.

Brouard, M., Duxon, S. P., Enriquez, P. A., and Simons, J. P., 1992, J. chem. Phys., 97, 7414.

Busch G. E., and Wilson K., 1972, J. chem. Phys., 56, 3626.

Cavero, V., L’Hermite, J. M., Rahmat, G., and Vetter, R., 1999, J. chem. Phys., 110, 3428.

Chen, J. J., Hung, Y. M., Liu, D. K., Fung, H. S., and Lin, K. C., 2001, J. chem. Phys., 114, 9395.

Chen, M. L., Lin, W. C., and Luh, W. T., 1997, J. chem. Phys., 106, 5972.

Child, M. S., 1974, Molecular Collision Theory (New York: Academic Press).

Crépin, C., Picqué, J. L., Rahmat, G., Vergès, J., Vetter, R., Gadéa, F. X., Pélissier, M., Spiegelmann, F., and Malrieu, J. P., 1984, Chem. Phys. Lett., 110, 395.

Cruse, H. W., Dagdigian, P. J., and Zare, R. N., 1973, Discuss. Faraday Soc., 55, 277.

Davies, H. F., Suits, A. G., Hou, H., and Lee, Y. T., 1990, Ber. Bunsenges phys. Chem., 94, 1193.

De Pujo, P., Sublimontier, O., Visticot, J. P., Berlande, J., Cuvellier, J., Alcaraz, C., Gustavsson, T., Mestdagh, J. M., and Meynadier, P., 1993, J. chem. Phys., 99, 2533.

Demtroder W., 1988, Atomic and Molecular Beam Methods, Vol. II, edited by G. Scoles (Oxford University Press), p. 213.

Fan, L. H., Chen, J. J., Lin, Y. Y.., and Luh, W. T., 1999, Phys. Chem. A, 103, 1300.

Fluendy, M. A. D., and Lawley, K. P., 1973, Chemical Applications of Molecular Beam Scattering (London: Chapman \& Hall).

Freidrich, B., and Herschbach, D. R., 1991, Z. Phys. D, 18, 153.

GadéA, F. X., and Durup, J., 1987, Chem. Phys. Lett., 138, 43.

Gadéa, F. X., L’Hermite, J. M., Rahmat, G., and Vetter, R., 1988, Chem. Phys. Lett., $151,183$.

Gadéa, F. X., Spiegelmann, F., Pélissier, M., and Malrieu, J. P., 1986, J. chem. Phys., 84, 4872.

Gandhi, S. R., Curtiss, T. J., Xu, Q. X., Choi, S. E., and Bernstein, R. B., 1986, Chem. Phys. Lett., 132, 6.

Gaubatz, U., Rudecki, P., Becker, M., Schiemann, S., Kulz, M., and Bergmann, K., 1989, Chem. Phys. Lett., 149, 463.

Gerrity, D. P., and Valentini, J. J., 1983, J. chem. Phys., 79, 5202; 1985, ibid., 82, 1323.

Gianturco, F. A., Buonomo, E., Kumar, S., Clarke, N. J., Sironi, M., and Raimondi, M., 1998, Chem. Phys., 230, 350.

Gianturco, F. A., Bodo, E., Martinazzo, R., and Raimondi, M., 2001, Eur. Phys. J., D15, 321 .

Gillen, K. T., Rulis, A. M., and Bernstein, R. B., 1971, J. chem. Phys., 54, 2831.

Gonzalez-Urena, A., and Vetter, R., 1993, Comments at. molec. Phys. D, 29, 97; 1995, J. chem. Soc., Faraday Trans., 91, 389; 1996, Int. Rev. phys. Chem., 15, 375.

Gotting, R., Mayne, H. R., and Toennies, J. P., 1984, J. chem. Phys., 80, 2230; 1986, Recent Advances in Molecular Reaction Dynamics, edited by R. Vetter and J. Vigué (Paris: Editions du CNRS), p. 85.

Grice, R., and Empedocles, P. B., 1968, J. chem. Phys., 48, 5352.

Hefter, U., and Bergmann, K., 1988, Atomic and Molecular Beam Methods, Vol. I, edited by G. Scoles (Oxford University Press), p. 193.

Hering, P., Brooks, P. R., Curl, R. F., Jodson, R. S., and Lowe, F. S., 1980, Phys. Rev. Lett., 44, 687.

Herschbach, D. R., 1965, Appl. Optics Suppl., 2, 128; 1966, Adv. chem. Phys., 10, 319; 1987, Les Prix Nobel 1986 (Stockholm: Almquist \& Wiksell).

Hertel, I. V., 1982, Dynamics of the Excited State, Advances in Chemical Physics, edited by K. P. Lawley (New York: Wiley), p. 475. 
Hertel, I. V., Hofman, H., and Rost, K. A., 1977, Phys. Rev. Lett., 38, 343.

Hertel, I. V., and Storl, W., 1978, Adv. at. molec. Phys., 13, 113.

Hirst, D. M., 1985, Potential Energy Surfaces (London: Taylor and Francis).

Hsiao, Y. C., Liu, D. K., Fung, H. S., and Lin, K. C., 2000, J. chem. Phys., 113, 461.

Huang, X., ZhaO, J., XIng, G., Wang, X., and Bersohn, R., 1996, J. chem. Phys., 104, 1338.

Huber, J. R., and Schinke, R., 1993, J. phys. Chem., 97, 3463.

Hung, Y.M., and Lin, K.C., 2001, J. phys. Chem. A, 105, 41.

Jeung, G. H., Lee, H. S., Kim, K. H., and Lee, Y. S., 2002, Phys. Lett., 358, 151.

Jouvet, C., and SoeP, B., 1984, J. chem. Phys., 80, 2229.

Jouvet, C., Soep, B., Breckenrdige, W. H., Whitham, C., and Visticot, J. P., 1989, J. chem. Soc., Faraday Trans., 85, 113.

Kim, K. H., Lee, H. S., Lee, Y. S., and JeUng, G. H., 2002, J. chem. Phys., 116, 589.

Kinsey, J. L., 1977, J. chem. Phys., 66, 2560.

Kleiber, P. D., Stwalley, W. C., and Sando, K. M., 1993, A. Rev. phys. Chem., 44, 13.

Kramer, K. H., and Bernstein, R. B., 1965, J. chem. Phys., 42, 767.

Kuntz, P. J., Mok, M. H., and Polanyi, J. C., 1969, J. chem. Phys., 50, 4623.

Kuntz, P. J., Nemeth, E. M., and Polanyi, J. C., 1969, J. chem. Phys., 50, 4607.

Kuppermann A., 1981, Theoretical Chemistry: Theory of Scattering (New York: Academic Press), vol. 6, A, p. 79.

Launay, J. M., Le Dourneuf, M., and Lepetit, B., 1986, Recent Advances in Molecular Reaction Dynamics, edited by R. Vetter and J. Vigué (Paris: Editions du CNRS), p. 163.

L'Hermite, J. M., 1992, J. chem. Phys., 97, 6215.

L'Hermite, J. M., Rahmat, G., and Vetter, R., 1990a, Laser Chem., 10, 1990; 1990b, J. chem. Phys., 93, 434; 1991, ibid., 95, 3347.

Lee, H. S., LeE, Y. S., and Jeung, G. H., 1999, J. phys. Chem. A, 103, 11080.

Lee, Y. T., 1987, Les Prix Nobel 1986 (Stockholm: Almquist \& Wiksell), p. 168.

Lehr, L., Motzkus, M., Pichler, G., and Hering, P., 1998, J. Raman Spectrosc., 29, 273.

Leone, S. R., 1988, Selectivity in Chemical Reactions, edited by J. C. Whitehead (Dordrecht: Kluwer), p. 245.

Lepetit, B., Launay, J. M., and Le Dourneuf, M., 1989, Chem. Phys., 134, 1.

Levine, R. D., and Bernstein, R. B., 1987, Molecular Reaction Dynamics and Chemical Reactivity (Oxford University Press).

Lin, K. C., and Chang, H. C., 1989, J. chem. Phys., 90, 6151.

Liu, D. K., Chen, J. J., Nien, C. F., and Lin, K. C., 1999, J. chem. Phys., 111, 5277.

Liu, D. K., and Lin, K. C., 1996, J. chem. Phys., 105, 9121; 1997, ibid., 107, 4244.

LiU, D. K., Lin, K. C., and Chen, J. J., 2000, J. chem. Phys., 113, 5302.

Loesch, H. J., and Reincheid, A., 1990, J. chem. Phys., 94, 4779; 1991, J. phys. Chem., 95, 8194.

Marinero, E. E., Rettner, C. T., and Zare, R. N., 1984, J. chem. Phys., 80, 4142.

Martinez, T., 1997, Chem. Phys. Lett., 272, 139.

Mayne, H. R., and Toennies, J. P., 1981, J. chem. Phys., 75, 1794.

Meier, W., Rottke, H., Zacharias, H., and Welghe, K. H., 1985, J. chem. Phys., 83, 4360.

Mestdagh, J. M., Balko, B. A., Covinsky, M. H., Weiss, P. S., Vernon, M. F., Schmidt, H., and LeE, Y. T., 1987, Discuss. Faraday Soc., 84, 145.

Mestdagh, J. M., Visticot, J. P., and Suits, A. G., 1994, The Chemical Dynamics and Kinetics of Small Radicals, edited by K. Liu and A. Wagner (Singapore: World Scientific), p. 668.

Menzinger, M., 1988, Selectivity in Chemical Reactions, edited by J. C. Whitehead (Dordrecht: Kluwer), p. 457.

Motzkus, M., Pichler, G., Kompa, K. L., and Hering, P., 1997, J. chem. Phys., 106, 9057; 1998, ibid., 108, 9291.

Murphy, E. J., Brophy, J. H., Arnold, G. S., Dimpfl, W. L., and Kinsey, J. L., 1979, J. chem. Phys., 70, 5910.

Mysers, E. G., Murnick, D. E., and Softky, W. R., 1987, Appl. Phys. B, 43, 247. 
Neumark, D. M., Wodkte, A. M., Robinson, G. N., Hayden, C. C., and Lee, Y. T., 1985a, J. chem. Phys., 82, 3045.

Neumark, D. M., Wodtke, A. M., Robinson, G. N., Hayden, C. C., Shobatake, K.,

Sparks, R. K., Schafer, T. P., and Lee, Y. T., 1985b, J. chem. Phys., 82, 3067.

Nieh, J. C., and Valentini, J. J., 1988, Phys. Rev. Lett., 60, 519; 1990, J. chem. Phys., 92, 1083.

Oberlander, M. D., Kampf, R. P., and Parson, J. M., 1991, Chem. Phys. Lett., 176, 385.

Ou, Y. R., Liu D. K., and Lin, K. C., 1998, J. chem. Phys., 108, 1475.

Parker, D. H., Chakravorty, K. K., and Bernstein, R. B., 1982, Chem. Phys. Lett., 86, 113.

Parson, J. M., Shobatake, K., Lee, Y. T., and Rice, S. A., 1973, Discuss. Faraday Soc., $55,344$.

Perry, D. S., and Polanyi, J. C., 1976, Chem. Phys., 12, 37.

Picqué, J. L., Vergès, J., and Vetter, R., 1980, J. Phys. (Paris), 41, L305.

Polanyi, M., 1932, Atomic Reactions (London: Williams and Norgate).

Polanyi, J. C., and Schreiber, J. L., 1974, Physical Chemistry, an Advance Treaty, edited by W. Jost (New York: Academic Press), p. 383.

Polanyi, J. C., and Woodall, K. B., 1972, J. chem. Phys., 57, 1574.

Prisant, M. G., Rettiner, C. T., and Zare, R. N., 1984, J. chem. Phys., 81, 2699.

Rahmat, G., Spiegelmann, F., Vergès, J., and Vetter R., 1987, Chem. Phys. Lett., 135, 459.

Rahmat, G., Vergès, J., Vetter, R., Gadéa, F. X., Pelissier, M., and Spiegelmann, F., 1986, Recent Advances in Molecular Reaction Dynamics, edited by R. Vetter and J. Vigué (Paris: Editions du CNRS), p. 225.

Rettner, C. T., and Zare, R. N., 1981, J. chem. Phys., 74, 3630; 1982, ibid., 77, 2416.

Rinnen, K. D., Kliner, D. A. V., and Zare, R. N., 1989, J. chem. Phys., 91, 7514.

Rossi, F., and Pascale, J., 1985, Phys. Rev. A, 32, 2657.

Rulis, A. M., and Bernstein, R. B., 1972, J. chem. Phys., 57, 5497.

Sayer, B., Ferray, M., Lozingot, L., and Berlande, J., 1981, J. chem. Phys., 75, 1894.

SChaefer III, H. T., 1977a, Methods of Electronic Structure Theory, Vol. 3 (New York: Plenum); 1977b, Modern Theoretical Chemistry, Vol. 4 (New York: Plenum).

Scherer, N. F., Khundkar, L. R., Bernstein, R. B., and Zewail, A. H., 1987, J. chem. Phys., 87, 145.

Schiemann, S., Kahm, S., Steuerwald, S., and Bergmann, K., 1993, Phys. Rev. Lett., 71, 3637.

Schinke, R., 1989, Comments at. molec. Phys., 23, 15; 1993, Photodissociation Dynamics (Cambridge University Press).

Scoles, G. (editor), 1988, Atomic and Molecular Beam Methods, Vols I, II (Oxford University Press).

Serri, J. A., Kinsey, J. L., and Pritchard, D. E., 1981, J. chem. Phys., 75, 663.

Sevin, A., and Chaquin, P., 1985, Chem. Phys., 93, 49.

Skenderovic, H., Ban, T., and Pichler, G., 2000, J. Phys. D., 33, 396.

Smith, I. W., 1980, Kinetics and Dynamics of Elementary Gas Reactions (London: Butterworths).

Stancil, P. C., LePp, S., and Dalgarno, A., 1996, Astrophys. J., 458, 397.

Stolte, S., Chakravorti, K. K., Bernstein, R. B., and Parker, D. H., 1982, Chem. Phys., 71, 353.

Suits, A. G., Bontuyan, L. S., Houston, P. L., and Whitaker, B. J., 1992, J. chem. Phys., 96, 8618.

Tam, A., Moe, G., and Happer, W., 1975, Phys. Rev. Lett., 35, 1630.

Toennies, J. P., 1974, Physical Chemistry, an Advance Treaty, edited by W. Jost (New York: Academic Press), p. 228.

Truhlar, D. G., and Muckerman, J. T., 1979, Atom-Molecule Collision Theory: A Guide for the Experimentalist, edited by R. B. Bernstein (New York: Plenum).

Tsukiyama, K., Kata, M. B., and Bershohn, R., 1980, J. chem. Phys., 84, 1934.

Van der Zande, W. J., Zhang, R., Zare, R. N., MacKendrick, K. G., and Valentini, J. J., 1991, J. phys. Chem., 95, 8205. 
Valentini, J. J., Lee, Y. T., and Auerbach, D., 1977, J. chem. Phys., 67, 4866.

Veirs, D. K., Rosenblatt, G. M., and Valentini, J. J., 1985, J. chem. Phys., 83, 1605.

Vernon, M. F., Schmidt, H., Weiss, P. S., Covinski, M. H., and Lee, Y. T., 1986, J. chem. Phys., 84, 5580.

Visticot, J. P., Ferray, M., Lozingot, L., and Sayer, B., 1983, J. chem. Phys., 79, 2839.

Weiss, P. S., Mestdagh, J. M., Schmidt, H., Vernon, M. F., Covinski, M. H., Balko, B. A., and LeE, Y. T., 1986, Recent Advances in Molecular Reaction Dynamics, edited by R. Vetter and J.Vigué (Paris: Editions du CNRS), p. 15.

Zare, R. N., 1972, Molec. Photochem., 4, 1; 1977, Scient. Am., 236, 86; 1979, Discuss. Faraday Soc., 67, 7; 1988, Angular Momenta (New York: Wiley). 
Copyright of International Reviews in Physical Chemistry is the property of Taylor \& Francis Ltd and its content may not be copied or emailed to multiple sites or posted to a listserv without the copyright holder's express written permission. However, users may print, download, or email articles for individual use. 